Received Date : 06-May-2015

Revised Date : 06-Oct-2015

Accepted Date : 10-Oct-2015

Article type : Research Report

\title{
Network-selectivity and stimulus-discrimination in the primary visual cortex: cell-assembly dynamics
}

\author{
Vishal Bharmauria ${ }^{1,2}$, Lyes Bachatene ${ }^{1,2}$, Sarah Cattan $^{1,2}$, Simon Brodeur ${ }^{2,3}$, Nayan \\ Chanauria $^{1,2}$, Jean Rouat ${ }^{1,2,3}$ and Stéphane Molotchnikoff ${ }^{1,2,3 *}$
}

${ }^{1}$ Neurophysiology of Visual System, Université de Montréal, Département de Sciences Biologiques, Montréal, Qc, Canada

${ }^{2}$ Neurosciences Computationnelles et Traitement Intelligent des Signaux (NECOTIS),

Sherbrooke, Qc, Canada

${ }^{3}$ Département de Génie Électrique et Génie Informatique, Université de Sherbrooke, Sherbrooke, Qc, Canada

\section{Correspondence:}

Dr. Stéphane Molotchnikoff

Dépt. Sciences Biologiques

Université de Montréal

CP 6128 Succursale Centre-Ville

Montreal, H3C 3J7, PQ, Canada

stephane.molotchnikoff@umontreal.ca

Keywords: functional connection, network-selectivity, stimulus-discrimination, primary visual cortex, assembly-dynamics

This article has been accepted for publication and undergone full peer review but has not been through the copyediting, typesetting, pagination and proofreading process, which may lead to differences between this version and the Version of Record. Please cite this article as doi: 10.1111/ejn.13101

This article is protected by copyright. All rights reserved. 


\begin{abstract}
Visual neurons coordinate their responses in relation to the stimulus; however, the complex interplay between a stimulus and the functional dynamics of an assembly still eludes neuroscientists. To this aim, we recorded cell assemblies from multi-electrodes in the primary visual cortex of anaesthetized cats in response to randomly presented sine-wave drifting gratings whose orientation tilted in $22.5^{\circ}$ steps. Cross-correlograms divulged the functional connections at all the tested orientations. We show that a cell-assembly discriminates between orientations by recruiting a 'salient' functional network at every presented orientation, wherein, the connections and their strengths (peak-probabilities in the cross-correlogram) change from one orientation to another. Within these assemblies, closely tuned neurons exhibited increased connectivity and connection-strengths than differently tuned neurons. Minimal connectivity between untuned neurons suggests the significance of neuronal selectivity in assemblies. This study reflects upon the dynamics of functional connectivity, and brings to the fore the importance of a 'signature' functional network in an assembly that is strictly related to a specific stimulus. Apparently, it points to the fact that an assembly is the major 'functional unit' of information processing in cortical circuits, rather than the individual neurons.
\end{abstract}

\title{
Introduction
}

'Connectomics' which refers to the mapping of the neuronal circuits, is an emerging field in neurosciences, and it promises significant insights into the brain function and dynamics (Sporns et al., 2005; Hagmann et al., 2010; Sporns, 2011; Alivisatos et al., 2012; Leergaard et al., 2012). The developing consensus is that microcircuits (synonymous to sub-networks, cell-assemblies, coactive neurons, synfire chains, clicks, songs, ensembles) are fundamental to information

This article is protected by copyright. All rights reserved. 
processing in brain (Buzsáki , 2010; Harris \& Mrsic-Flogel, 2013); yet, the knowledge about their functional dynamics in relation to the sensory environment, is still sparse.

Many reports document that the mere co-activation of a neuronal group is not an evidence of the functional interactions between them (Kenet et al., 2003; Han et al., 2008; Mohajerani et al., 2013; Miller et al., 2014). Neurons in primary visual cortex (V1) are exquisitely selective to a preferred edge of an orientation when it is presented within their receptive fields (Hubel \& Wiesel, 1959; Bishop \& Henry, 1972). In cats, V1 neurons are columned into homogeneous populations with similar response properties; yet, they exhibit abundant anatomical and functional connections with neurons in adjacent columns (DeAngelis et al., 1999; Kisvárday et al., 2000; Binzegger et al., 2004; Stepanyants et al., 2008; Martin \& Schröder, 2013). Albeit, V1 has been studied for decades, the functional interplay between an assembly and the applied orientation is still not completely understood. Thereof, we sought to examine how an orientationselective assembly in cat-V1 (layer II/III, area 17) engineers functional connections between its neurons in response to different orientations.

In parallel to the neuronal selectivity (Butts \& Goldman, 2006), it has been shown that encoding sensory stimuli may be achieved by decoding sparsely firing neurons within assemblies that display time-relationships (functional synchrony) between their respective spike trains (Csicsvari et al., 1998; Barthó et al., 2004; Fujisawa et al., 2008; Bharmauria et al., 2014; Schwindel et al., 2014; König et al., 1995) within the rhythmic oscillations of the brain (Gray \& Singer, 1989; Engel et al., 2001; Fries et al., 2007; Bharmauria et al., 2015). Traditionally, cross-correlogram (CCG) analysis is an effective tool to reveal these functional causalities that are indicative of

This article is protected by copyright. All rights reserved. 
putative synaptic contacts between neurons (Perkel et al., 1967; König et al., 1995; Csicsvari et al., 1998; Shannon et al., 2000; Barthó et al., 2004; Fujisawa et al., 2008; Vizuete et al., 2012; Bharmauria et al., 2014; Schwindel et al., 2014).

Employing CCG strategy, we report that a salient functional network is activated within a cellassembly contingent upon the presented orientation, wherein connections are maintained, activated or inactivated with changing strengths as the orientation changes. Thus, along with our previous work (Bharmauria et al., 2014; Bharmauria et al., 2015), we suggest that an assembly is synergistically engaged within gamma oscillations in a characteristical 50-ms temporal 'window of opportunity' to frame a specific functional network in relation to the stimulus.

In this investigation, we systematically presented oriented sine-wave gratings in $22.5^{\circ}$ steps to examine the functional modulation (peak-modulation in CCG) in assemblies in relation to the applied axis of orientation, unlike previous reports (Kenet et al., 2003; Ecker et al., 2010; Talebi \& Baker, 2012; Miller et al., 2014; Martin \& Schröder, 2013), wherein they compared two very dissimilar stimuli (e.g. gratings vs. natural images or gratings vs. no stimulus). As far as we know, this study is the first to show the dynamic formation of a unique functional network within an assembly in response to the systematic change of an input stimulus. In short, this work demonstrates that network-selectivity within an assembly is the underpinning of stimulusdiscrimination in cortical circuits.

This article is protected by copyright. All rights reserved. 


\section{Materials and methods}

\section{Ethical approval}

Nine adult cats (weight: $2.0-3.0 \mathrm{~kg}, \sim 12-24$ months) of either sex were prepared for electrophysiological recordings in the primary visual cortex, as per the guidelines of Canadian Council on Animal Care. The protocol was approved by the Institutional Animal Care and Use committee of Université de Montréal. The full description of the procedure is given below.

\section{Animal, anesthesia and surgical procedures}

Briefly, cats were premedicated with acepromazine maleate (Atravet, Wyeth-Ayerst, Guelph, Ontario, Canada; $1 \mathrm{mg} \cdot \mathrm{kg}^{-1}$, intramuscular) and atropine sulphate (ATRO-SA, Rafter, Calgary, Canada; $0.04 \mathrm{mg} \cdot \mathrm{kg}^{-1}$, intramuscular), and anesthetized with ketamine hydrochloride (Rogarsetic, Pfizer, Kirkland, QC, Canada; $25 \mathrm{mg} \cdot \mathrm{kg}^{-1}$, intramuscular). Lidocaine hydrochloride (2\% Xylocaine, AstraZeneca, Mississauga, Ontario, Canada) was administered subcutaneously for local anesthesia at the surgical site. For artificial ventilation, a tracheotomy was performed and during the surgery the cats were sustained using 2\% isoflurane (AErrane, Baxter, Toronto, ON, Canada) ventilation. Xylocaine gel (5\%; Astra Pharma, Mississauga, Ontario, Canada) was applied at the pressure points. Tribrissen $\left(30 \mathrm{mg} \cdot \mathrm{kg}^{-1} \cdot \mathrm{day}^{-1}\right.$, subcutaneous; Schering-Plough, Point-Claire, Quebec, Canada) and Duplocillin (0.1 mL. $\mathrm{kg}^{-1}$, intramuscular; Intervet, Whitby, Ontario, Canada) were administered to prevent infection. After the surgery, the cats were fixed in a stereotaxic apparatus. Paralysis was induced with $40 \mathrm{mg}$ of gallamine triethiodide and maintained with $10 \mathrm{mg} \cdot \mathrm{kg}^{-1} \cdot \mathrm{h}^{-1}$ gallamine triethiodide (Flaxedil, Sigma Chemical, St. Louis, MO, USA; intravenous) administered in 5\% dextrose lactated Ringer's nutritive solution. General anesthesia was maintained by artificial ventilation with a mixture of $\mathrm{N}_{2} \mathrm{O} / \mathrm{O}_{2} \quad(70: 30)$

This article is protected by copyright. All rights reserved. 
supplemented with $0.5 \%$ isoflurane. Electroencephalogram, electrocardiogram, rectal temperature (using a heated pad to maintain a body temperature of $37.5{ }^{\circ} \mathrm{C}$ ) and end-tidal $\mathrm{CO}_{2}$ partial pressure $(25-30 \mathrm{mmHg})$ were monitored throughout the experiment, and kept in physiological ranges. The pupils were dilated with atropine sulfate (1\%, Isopto-Atropine; Alcon, Mississauga, Ontario, Canada) and the nictitating membranes were retracted with phenylephrine hydrochloride (2.5\%, Mydfrin, Alcon). The loci of the area centrales were inferred from the position of the blind spots which were opthalmoscopically focused and projected onto a translucent screen. Plano lenses with artificial pupils ( $5 \mathrm{~mm}$ diameter) were fixed on the cat's eyes to prevent the cornea from drying and to improve the acuity. At the end of the experiment, the cats were euthanized intravenously with a dose $\left(0.5 \mathrm{~mL} \cdot \mathrm{kg}^{-1}\right)$ of Sodium Pentobarbital (CEVA, Sante Animale).

\section{Visual stimulation, electrophysiological recording and single-unit selection}

Monocular stimulation was done. A schematic of the experimental setup is shown in Fig. 1. The multi-unit receptive fields (RF) were mapped as the minimum response field (Barlow et al., 1967) by using a hand-held ophthalmoscope after clearly detectable activity was obtained. These preliminary tests revealed qualitative properties such as dimensions, velocity preference, orientation and directional selectivity of neurons. Thereafter, the visual stimuli were generated with a VSG 2/5 graphic board (Cambridge Research Systems, Rochester, England) and displayed on a 21-inch monitor (Sony GDM-F520 Trinitron, Tokyo, Japan) placed $57 \mathrm{~cm}$ from the cat's eyes, with $1024 \times 768$ pixels, running at $100-\mathrm{Hz}$ frame refresh. The blank screen was uniformly gray $\left(\sim 35 \mathrm{~cd} \cdot \mathrm{m}^{-2}\right)$. Contrast and mean luminance were set at $80 \%$ and $40 \mathrm{~cd} \cdot \mathrm{m}^{-2}$ respectively.

Optimal spatial and temporal frequencies were set at 0.24 cycles.deg ${ }^{-1}$ and in the range of

This article is protected by copyright. All rights reserved. 
1.0-2.0 Hz respectively, where V1 neurons are driven maximally by sine-wave drifting gratings (Bardy et al., 2006). The tested orientations were presented in a random order. Each drifting grating was presented in blocks of 25 trials (each trial lasted $4.1 \mathrm{~s}$ ) with varying inter-stimulus (1-3 s) intervals during which no stimulus was presented (Fig. 1A). Thus the presentation of a stimulus lasted $180 \mathrm{~s}$ (with all trials and inter-stimulus intervals).

Multi-unit activity in the primary visual cortex (layer II/III, area 17) was recorded using tungsten multi-electrode (Frederick Haer \& Co, Matrix Electrode) that had four columns (inter-column distance equals $410 \mu \mathrm{m}$ ) and each column had one row. For each recording, only two contacts were used as shown in Fig. 1B (spaced by 410 or $820 \mu \mathrm{m}$ ). The excitatory RF's (sine-wave drifting grating covering the excitatory RF of neurons at both electrodes) extended from $4^{\circ}-5^{\circ}$. The average overlap for the excitatory RF was $81 \pm 11 \%$. The RF's were located centrally within a $15^{\circ}$ radius from fovea. Twenty four recordings were done across all cats in the supragranular layers (cortical depth $<1000 \mu \mathrm{m}$; mean $=650 \mu \mathrm{m}$ ), either in the left or the right hemisphere. Twenty two recordings were tested once; two recordings were tested twice, i.e., the same stimulus was tested after $\sim 1 \mathrm{hr}$. The signal from the microelectrodes was amplified, band-pass filtered (300 Hz-3 kHz), digitized and recorded with a $0.05 \mathrm{~ms}$ temporal resolution (Spike2, CED, Cambridge, England). Spike sorting from the multi-unit activity was performed, and each microelectrode yielded isolated single units (Fig. 1C). The units were discriminated on the basis of three criteria: 1) the spike-waveform difference 2) principal component analysis (PCA) showing well dissociated clusters 3) and auto-correlograms (ACG) with a characteristic refractory period (no events at zero) indicating the absence of contamination (Csicsvari et al., 1998; Barthó et al., 2004; Fujisawa et al., 2008; Bharmauria et al., 2014; Denman \& Contreras, 
2014). The stability of each cell's activity across conditions was verified qualitatively by the visual control of the disposition of clusters and the shapes of waveforms. Cluster-analysis was done using Spike2, CED, Cambridge, England in a 3-dimensional plot. The isolation distance was calculated as the Mahalanobis distance. Mahalanobis distance is the distance from the cluster center within which as many events belong to the other clusters as many belong to the specified cluster (Harris et al., 2001). In other words, given the multivariate data values for which the values in each variable are normally distributed around a mean, this measure allows to define boundaries of constant probability around the multi-dimensional center of the distribution. Thus, this estimation allows the separation of a cluster from the nearest cluster. Units with Mahalanobis distance greater than 2.5 were considered for the further analysis of the spike trains to reveal the functional connections between them. Once the clusters were computed using Mahalanobis distance, J3 values for neuron pairs were calculated indicating the distance separating the clusters.

where $\mathrm{J} 3=\mathrm{J} 2 / \mathrm{J} 1$

where, $\mathrm{J} 2$ equals the sum of the squared distances of all the clustered points from the centroid of all the points; and $\mathrm{J} 1$ equals the sum of the squared distances of each point from the centroid of the cluster it belongs to. A J3 value of 1.5 was set as benchmark for the separation (Bharmauria et al., 2015).

\section{Orientation tuning and OSI (Orientation Selectivity Index) computation}

Once single cells had been sorted out offline from multi-unit spike trains accumulated during data acquisition, orientation tuning curves of cells were obtained from the raw data and fitted with the von Mises function (Swindale, 1998).

This article is protected by copyright. All rights reserved. 
$M(\boldsymbol{\theta})=A \cdot e^{b(\cos (\Theta-c))}+\mathrm{d} \quad($ ii $)$

where, ' $A$ ' is the value of the function at the preferred orientation ' $c$ ', and ' $b$ ' is the width parameter. An additional parameter, 'd', represents the spontaneous firing rate of the cell. ' $M(\Theta)$ ' is the firing rate of the neuron at orientation, ' $\theta$ '. This allowed us to determine the preferred orientation of every cell. An orientation selectivity index (OSI) was calculated to ensure the tuning of neurons. It was measured using the fitted tuning curves by dividing the firing rate at baseline (orthogonal orientations) by the firing rate at the preferred orientation, and subtracting the result from one (Ramoa et al., 2001; Liao et al., 2004; Bachatene et al., 2013). The closer the OSI is to one, the stronger the orientation selectivity. It is to be underlined that within the context of this paper, we have considered neurons with OSI less than 0.5 as untuned neurons.

\section{Cross-correlograms (CCG) and Network-formation}

Cross-correlogram (CCG) is a histogram of the spikes of the target neuron in relation to the spikes of the reference neuron (spike-by-spike analysis). CCGs were computed (binwidth $=1$ ms) between the neural activities of all the possible neuron pairs at all the applied orientations to reveal the functional connections (Fig. 1D). The raw CCGs were shift-corrected to eliminate the putative significant peaks due to the simultaneous stimulation of both cells during each trial (to remove the stimulus evoked and locked components) (Perkel et al., 1967). A significant peak of $2 \mathrm{~ms}$ (two adjacent bins) or at least one significant bin (Alloway \& Roy, 2002) was searched within a window of $\pm 5 \mathrm{~ms}$ offset from zero (excluding the $\pm 1 \mathrm{~ms}$ bins around zero) in the shiftcorrected CCG to reveal a functional connection between two neurons. The statistical threshold

This article is protected by copyright. All rights reserved. 
for the significant peak was set at $95 \%$, and the probability of the neuron firing in a bin is calculated as follows (Abeles, 1982):

Considering the spike train is a Poisson process, the probability $(\mathrm{P})$ of a neuron's firing in the small bin of the size $b$ is:

$\mathrm{P}=\mathrm{F}^{*} \mathrm{~b}$

$\mathrm{F}=\mathrm{N} / \mathrm{T}$

where, ' $\mathrm{F}$ ' is the neuronal firing rate, ' $\mathrm{T}$ ' is the total time interval, and ' $\mathrm{N}$ ' is the number of spikes in that interval.

The expected bin count ' $C$ ' for the histogram is:

$\mathrm{C}=\mathrm{P}^{*} \mathrm{NRef} \quad(\mathrm{v})$

where, 'NRef' is the number of the reference events (spikes of the reference neuron, that is, at time zero in the CCG). We calculated 95\% intervals for ' $\mathrm{P}$ ' and 'C' according to Abeles, (1982).

A hypothetical network between three neurons (isolated from a microelectrode) at every orientation is displayed in Fig. 1E. For every CCG, the cumulative distribution of spikes was computed. This computation further indicates the modulation of the firing rate of the target cell after the reference cell fired an action potential. The cumulative sum (cs) graphs above histograms are calculated for each bin i as:

$c s[i]=\sum_{j=1}^{i} b c[j]-A^{i} ; \mathrm{i}=1,2, \ldots, \mathrm{N}$

where, 'bc' is the bin counts (i.e. the number of spikes falling inside the histogram bin ' $\mathrm{j}$ ') and 'A' the average of all 'bc'.

This article is protected by copyright. All rights reserved. 


\section{Discrimination Index (DI) Calculation}

The discrimination index (DI) for a cell-assembly was calculated as:

$\mathrm{DI}=\mathrm{N}^{\mathrm{s}} / \mathrm{N}^{\mathrm{t}} \quad($ vii $)$

where, $\mathrm{N}^{\mathrm{s}}=$ Number of significant connections found at an orientation, and $\mathrm{N}^{\mathrm{t}}=$ total number of possible connections if all the neurons in the group were to establish functional connections at that orientation.

\section{Results}

The main aim of our current investigation is to examine the functional relationships between neurons in a cell-assembly (it is to be underlined that within the context of this paper, neurons simultaneously recorded from a microelectrode are termed a cell-assembly) with orientation as the target property. We recorded data in a total of 44 sites (tested once) in the primary visual cortex (area 17, layer II/III) of anesthetized cats using a tungsten multi-electrode. Neurons were discriminated based on spike waveforms, ACGs and cluster analysis (see methods). In total, 174 neurons were identified as clean and other 79 neurons were discarded (if they did not fit any of the above criteria for isolation). The meta-analysis for OSI of all neurons is shown in Fig. 2. The mean OSI was found to be 0.74 . Functional connections were disclosed within a quasi-synchrony time-window in CCGs, i.e., $\pm 5 \mathrm{~ms}$ around zero (see above).

\section{Functional Network between the Closely Tuned Neurons}

Neurons in the cat visual cortex are organized into orientation columns, thus, it is expected that neurons belonging to a column respond in unison putatively implying the existence of specific connections between them (Shadlen \& Newsome, 1998; Stepanyants et al., 2008). Fig. 3A shows

This article is protected by copyright. All rights reserved. 
five well isolated single units (distinct waveforms with respective responses and orientation tuning curves computed from the firing levels at each orientation) from the tip of a tungsten multi-electrode. The individuality of neurons was further assured by their respective ACGs (Fig. 3B), principal component analysis (PCA) denoting the clear separation of clusters (Fig. 3C, waveforms superimposed as an inset), and J3 value for each pair (Fig. 3D) that represents the isolation distance between every cluster pair.

Thereafter, the corresponding activities of all the pairs (ten for the above assembly) were crosscorrelated to reveal the functional connections between them. Fig. 3E shows the functional network between these neurons (similarly tuned, $\sim 45^{\circ}$, see polar plots inside units) when an oriented sine-wave drifting grating of $22.5^{\circ}$ was presented within their receptive field. Orange arrows depict the functional connections as revealed by CCGs (Fig. 3F) and are indicative of time-locked activity between the neurons. Because neurons physically close together are densely innervated to each other (Gilbert \& Wiesel, 1989; Binzegger et al., 2004; Stepanyants et al., 2008; Kampa et al., 2011), therefore, these revealed time-relationships may be inferred as putative synaptic links between neurons (Barthó et al., 2004; Fujisawa et al., 2008; Bharmauria et al., 2014; Schwindel et al., 2014). Neuron 2 (reference) projects onto neuron 1 (target), as the CCG significantly peaks $(\mathrm{P}=2.5 \%)$ within $5 \mathrm{~ms}$ after the zero mark. The cumulative histogram (above CCG) of the discharge rate of the target cell further illustrates that once the reference neuron produced a spike; the firing rate of the target unit was considerably modulated, thus, signifying the functional link between neurons. Neurons 1 (reference) and 4 (target) are reciprocally connected to each other, implying the recurrent excitation between them (Schwindel et al., 2014). First, the target neuron excites the reference neuron with a probability of $2 \%$ and 
then after a brief period of inhibition from third neuron(s) (the trough at zero in the CCG) the reference neuron back-projects and excites the target neuron with a probability of $1.7 \%$. Neuron 4 receives two connections, which is an indication of convergent input from several neurons in the microcircuit. Similarly, other connections were revealed, and consequently the whole network was disclosed at the applied orientation within an assembly.

Analogically, this seems to be in parallel to what Singer has opined in his review (Singer, 2013) — if we consider the recorded coactive neurons (assembly) as a reservoir with randomly coupled neurons, then the network framed within the reservoir could be compared to a classifier (network) that is stimulus-specific and emerges within this recurrent network. Notably, most of the peaks in CCGs were broad (beyond $2 \mathrm{~ms}$ ) and were most likely caused by the shared input to the target neuron, because many orientations are represented on the dendritic branches of a neuron. However, the synaptic drive related to the applied orientation dominates over others (Jia et al., 2010; Bachatene et al., 2013; Wertz et al., 2015), and, moreover, it has been shown that the transient surge of the target neuron discharge is predominantly caused by the reference neuron (Bharmauria et al., 2014).

\section{Functional Network between the Differently Tuned Neurons}

Neurons in an orientation column exhibit anatomical and functional connections with neurons from adjacent columns (Kisvárday et al., 2000; Binzegger et al., 2004; Stepanyants et al., 2008); therefore, neurons recorded within a cortical subspace from an electrode might exhibit different orientation selectivity from each other. Fig. 4A illustrates four simultaneously recorded neurons (differently tuned — neurons 2 and 3 are tuned similarly to $\sim 67.5^{\circ}$; whereas neuron 1 and neuron

This article is protected by copyright. All rights reserved. 
4 are more responsive at $0^{\circ}$ and $90^{\circ}$ respectively) with distinctive waveforms, the respective visual responses, and orientation tuning curves calculated from the firing at each orientation. Fig. $4 \mathrm{~B}$ represents the cluster-separation of the dissociated spikes that are overlaid as an inset. The network between these neurons (in response to $45^{\circ}$ ) is shown in Fig. 4C. Fig. 4D illustrates the CCGs for all the functionally connected pairs. In addition to the functional link between similarly tuned neurons (neuron 3 projects onto neuron $2, \mathrm{P}=3.3 \%$ ), neuron 3 and neuron 1 which display dissimilar tunings are reciprocally connected to each other (neuron 1 projects onto neuron $3, \mathrm{P}=2.9 \%$; neuron 3 back-projects onto neuron $1 ; \mathrm{P}=4.9 \%$ ). The blue neuron (tuned at $0^{\circ}$ ) projects onto the cyan neuron (tuned at $67.5^{\circ}$ ) with a probability of $0.95 \%$. Neuron 4 which is tuned to $90^{\circ}$ did not exhibit any connection. In summary, this shows that an assembly of differently orientation-selective neurons may feature a stimulus-encoding network between them.

\section{Network-selectivity and assembly dynamics}

As mentioned above, many reports have shown that a group of coactive neurons constitute a cellassembly; however, how these neurons functionally connect with each other in response to different stimuli, is yet not fully explored. To this end, we systematically wired networks between coactive neurons (the assembly in Fig. 3) at every presented orientation and spontaneous activity (Fig. 5A). A unique network was activated at every presented orientation. A maximum of five connections were disclosed at $22.5^{\circ}$, and in relation to that, only three connections were revealed at the optimal orientation $\left(45^{\circ}\right.$, which elicited highest firing rates $)-$ two connections were maintained (purple to yellow and purple to green); the connection between the blue-yellow pair changed to unidirectional from bidirectional; and the connection between the blue-green pair was deactivated. The orange connections were mostly maintained in spite of

This article is protected by copyright. All rights reserved. 
the change in orientation (e.g. yellow-purple pair), whereas the green links were activated only at some orientations. The connection from the blue to the yellow neuron was reversed at $135^{\circ}$. No connection was observed at $0^{\circ}$ and spontaneous firing (last box).

Furthermore, the connections were characterized by change in their probabilities $(\mathrm{P})$ from one orientation to another as depicted in the connectivity matrices (Fig. 5B); e.g., the connection between purple-yellow pair $\left(\mathrm{P}=3 \%\right.$ at $22.5^{\circ}, \mathrm{P}=1.65 \%$ at $45^{\circ}$ and $\mathrm{P}=0.7 \%$ at $\left.90^{\circ}\right)$. This is suggestive of the changing synaptic weights between neurons (Alloway \& Roy, 2002), and also shows the network-dynamics of the assembly as the orientation changes. A typical example of a CCG peak-modulation with orientation for a neuron pair (blue-yellow, blue is reference) is shown in Fig. 5 C. In most cases $\left(45^{\circ}, 67.5^{\circ}, 90^{\circ}\right.$ and $\left.157.5^{\circ}\right)$, the peak was maintained but the probability changed $(\mathrm{P}=2.3 \% ; \mathrm{P}=0.7 \% ; \mathrm{P}=1.6 \%$; and $\mathrm{P}=2 \%$ respectively $)$. At $22.5^{\circ}$, the pair exhibited a bidirectional connection (blue to yellow, $\mathrm{P}=1.7 \%$; yellow to blue, $\mathrm{P}=2 \%$ ). The position of the peak is reversed at $135^{\circ}$ with $\mathrm{P}=0.95 \%$. At $0^{\circ}$ and $112.5^{\circ}$, no peak was observed. Irrespective of the fact that firing rates of the neurons changed from one orientation to another (see counts/bin on y-axis); significant CCGs emerged, although, with changing strengths.

Taken together, we may conclude that the activation of a specific functional network between coactive neurons (cell-assembly) is 'inevitable’ for feature encoding as suggested by Miller et al (2014) (intrinsically generated and visually evoked ensembles are similar in activation pattern of neurons with one major difference — that visually evoked stimuli are 'time-locked' to stimuli whereas the spontaneous ensembles repeat randomly). In short, this analysis shows the dynamics

This article is protected by copyright. All rights reserved. 
of functional connectivity and network-selectivity within an assembly in response to different orientations.

\section{Peak-probability $(P)$ is independent of the firing rates of neurons}

We then investigated if the peak-probability was related to the firing rate of the neurons involved in a connection (Fig. 6). Fig. 6A illustrates this relationship for the connections $(n=22)$ in an assembly (in Fig. 5). The horizontal regression line $\left(P=0.58 ; R^{2}=0.02\right)$ indicates that ' $\mathrm{P}$ ' is independent of the cumulative firing (total number of spikes collected over 25 trials between a pair) of a neuron pair. Even globally, when neuron pairs $(\mathrm{n}=101)$ across different assemblies were pooled randomly, no relation $\left(P=0.71 ; R^{2}=0.001\right)$ was observed between ' $\mathrm{P}$ ' and the cumulative firing of neurons, which is in line with previous reports suggesting that these changes in peak-probability are independent of the firing rate of neurons (Duret et al., 2006; Ghisovan et al., 2008; Rolls \& Treves, 2011). Because weakly firing neurons also exhibited connections, this implies that these neurons also have important role to play in encoding stimuli.

Further (Fig. 6C), we randomly pooled unconnected pairs $(\mathrm{n}=71)$ to compare their evoked cumulative firing with the connected pairs $(\mathrm{n}=101)$. The mean (with SEM) cumulative firings for the connected and unconnected pairs were found to be $9.61 \pm 0.65$ and $9.90 \pm 1.63$ respectively and were non-significantly different, i.e. almost equivalent (unpaired $t$-test, $P=$ 0.71). However, the firing of the same unconnected pairs was found to be significantly different (unpaired $t$-test, $P=0.002)$ at the evoked (see above) and spontaneous $(4.78 \pm 0.57)$ conditions (that is, when the responses of same neurons were elicited with stimulus exhibiting higher cumulative firings, yet displaying no connections). The connected and spontaneous classes were

This article is protected by copyright. All rights reserved. 
significantly different from each other too (unpaired $t$-test, $\mathrm{P}<0.0001$ ). Collectively, this latter analysis further suggests that irrespective of the fact that connected and unconnected pairs exhibited similar firing patterns, the specific interactions between connected neurons yield the precise temporal relationships between their activities. The functional connection is thus established in relation to the applied stimulus.

\section{Discrimination Index (DI) and Orientation Range}

We then asked whether the probability of finding connections is related to the orientation range of neurons in an assembly. To this aim, a discrimination index (DI, see methods) was calculated for all assemblies (numbered on X-axis) at every tested orientation (Fig. 7). A neuron is depicted as a solid circle (pink for tuned and black for untuned) with its preferred orientation (Y-axis). The orientation range of an assembly is underlined by a black vertical line (for presentation, the smaller ranges are positioned to the left of the figure). In line with previous studies (Shadlen \&

Newsome, 1998; Ko et al., 2011; Ko et al., 2013; Cossell et al., 2015; Wertz et al., 2015), neurons in smaller ranges (assemblies 1-6) are frequently connected (even at different orientations), thus exhibiting higher DI (up to 1, see the color scale that stands for the DI, the DI values increase from blue to red) values. Assemblies \#7 and \#8 neurons display tight tuning; however, remain unconnected, suggesting that the selection of a functional network transcends their mere co-activation (Miller et al., 2014). As the range increased, there was a tendency for neurons to remain unconnected (low DI); however, assemblies (e.g., \#21, \#26, \#37) with larger orientation ranges $\left(\sim 75-150^{\circ}\right)$ also exhibited abundant functional connections (high DI). The higher the DI at a specific orientation, the least selective the assembly is for that stimulus property (e.g., assembly \#3 neurons that are closely tuned, have higher DI at all the orientations,

This article is protected by copyright. All rights reserved. 
and are, therefore, less selective and frequently connect to form a network); whereas, the lower the DI, the more selective the network is. Finally, untuned neurons did not exhibit many connections (e.g., assembly \#44 neurons with OSI < 0.5), suggesting that connectivity is largely dependent on orientation selectivity. Conclusively, this analysis shows that even though neurons in small-range assemblies (as if biased toward a particular orientation) connect more frequently with each other, yet, and importantly, high-range assemblies do exhibit connectivity too. It is to be noticed that newly active cells (six in number) which are not shown in the figure were also considered for calculating the DI at that specific orientation. Please see Fig. 8 for analysis on cell pair basis.

\section{Global Distribution of Connections within assemblies}

In total, 399 connections were found across all assemblies - 378 (236 unidirectional and 71 bidirectional, Fig. 8A) at all orientations (stimulus-locked) and 21 at spontaneous firing. It is to be underlined that a bidirectional connection consisted of two connections. The proportions of stimulus-locked (0.19) and spontaneously active (0.08) connections are significantly different $(z-$ test, $P<0.00001$ ) from each other (Fig. 8B), thus implying that the disclosed connections are strongly linked to stimuli.

Indeed, the DI computation (Fig. 7) hinted at the general distribution of connections, but to further investigate whether connections were linked to the orientation spread of involved neurons, we computed the absolute number of connections in assemblies as a function of their orientation spread (Fig. 8C, UT group was eliminated from this analysis). We found that no such relation (ANOVA, $P=0.49$ ) exists, suggesting that all assemblies tend to exhibit numerous

This article is protected by copyright. All rights reserved. 
connections. Because an orientation spread comprises neurons with different tunings, one may assume that closely tuned neurons in an assembly might exhibit more connectivity between them. Thus, further analysis was targeted to infer connectivity on neuron pair basis as a function of the orientation spread. The linear regression (Fig. 8D) showed a declining trend $\left(R^{2}=0.96, P\right.$ $=0.12$ ) for the proportion of connections (mean; 0.25 at $\pm 22^{\circ}, 0.19$ at $\pm 44^{\circ}, 0.16$ at $\pm 66^{\circ}$ ) as the spread increased until $\pm 66^{\circ}$ (it is to be underlined that, though the analysis was performed for $\pm 88^{\circ}$ but this spread was excluded from statistical tests for this and all subsequent analysis, as neurons involved in the pair were tuned to opposite directions). The proportion increased to 0.27 at $\pm 88^{\circ}$. Interestingly, in a similar way, the peak-probabilities $(\mathrm{P})$ also declined (mean \pm SEM; $2.65 \pm 0.17 \%$ at $\pm 22^{\circ} ; 2.02 \pm 0.17 \%$ at $\pm 44^{\circ}$ and $1.99 \pm 0.23 \%$ at $\left.\pm 66^{\circ}\right)\left(R^{2}=0.78, P=\right.$ 0.30 ) as a function of the orientation spread (Fig. 8E). The strength increased to $2.65 \pm 0.42 \%$ at $\pm 88^{\circ}$. Furthermore, the proportion $(0.23)$ of connected tuned pairs (T-T) was found to be significantly more (z-test, $P<0.00001)$ than the proportion $(0.025)$ of connections where an untuned (UT) neuron was involved in a connection with any other neuron (UT or T). In short, the above analyses point to the fact that neuronal selectivity is fundamental to functional connectivity, and closely tuned neurons connect more vigorously with each other than distantly tuned neurons in V1 microcircuits as reported previously (Stepanyants et al., 2008; Ko et al., 2011; Ko et al., 2013; Cossell et al., 2015). However, the high connectivity and increased strength of connections at $\pm 88^{\circ}$ could be attributed to specific connectivity pattern between neurons, as they have to process the contrasting features in the image.

This article is protected by copyright. All rights reserved. 
Thereafter, we analyzed the proportion of bidirectional and unidirectional connections in relation to the orientation spread (Fig. 8G). The unidirectional connections decreased from 0.15 to 0.14 to 0.12 at $\pm 22^{\circ}$ to $\pm 44^{\circ}$ to $\pm 66^{\circ}$ respectively, whereas the corresponding proportions for bidirectional connections were found to be $0.09,0.04$ and 0.04 . The ratio of bidirectional connections was significantly different between $\pm 22^{\circ}$ and $\pm 44^{\circ}$ ( $z$-test, $P=0.002$ ); and between $\pm 22^{\circ}$ and $\pm 66^{\circ}$ too ( $z$-test, $P=0.012$ ), however, it was found to be insignificant between $\pm 44^{\circ}$ and $\pm 66^{\circ}$ ( $z$-test, $P=0.48$ ). The corresponding analyses for unidirectional connections between all the classes did not exhibit significant differences between them ( $z$-test, $P>0.05$ ). The $\pm 88^{\circ}$ had a proportion of 0.12 and 0.14 unidirectional and bidirectional connections respectively. Finally (Fig. $8 \mathrm{H}$ ), the strength of the bidirectional connections (mean \pm SEM; $3.04 \pm 0.25 \%$ ) was found to be significantly (unpaired $t$-test, $P=0.0007$ ) higher than the unidirectional connections $(2.16 \pm 0.12 \%)$. In brief, the bidirectional connections are indicative of strong recurrent excitation between neuronal circuits, suggesting that recurrent excitation between neurons in an assembly plays a pivotal role in feature encoding as has been reported in literature (Bopp et al., 2014; Schwindel et al., 2014; Cossell et al., 2015). It is to be underlined that the calculations for the bidirectional connections were performed over all possible connections representing bidirectional connections (that is, if $\mathrm{X}$ represents a bidirectional connection, then the calculations were done over $2 \mathrm{X}$ ).

\section{Connections with distance}

Lateral connectivity is a characteristic feature of visual neurons (Shadlen \& Newsome, 1998), thus, further we sought to examine the connections between simultaneously recorded neurons from two microelectrodes (410 or $820 \mu \mathrm{m}$ apart). A typical example of neuronal connectivity

This article is protected by copyright. All rights reserved. 
between electrodes (spaced laterally by $820 \mu \mathrm{m}$ ) is shown in Fig 9A. CCG analysis revealed that the red neuron recorded from electrode-1 projects (blue arrow) onto the blue neuron recorded from electrode-2. In a similar fashion, the pink neuron recorded from electrode- 2 projects onto the purple neuron recorded from electrode-1. The green stars beside the neurons (purple from electrode-1 and blue from electrode-2) are indicative of synchrony between these neurons (peak straddling zero in the $\mathrm{CCG}$ ) and presumably receiving a common afferent input from a third neuron (König et al., 1995; Ghisovan et al., 2008; Denman \& Contreras, 2014). It is to be mentioned that a single orientation grating was positioned on the monitor screen covering the receptive fields of neurons at both sites. In total, only 48 connections (37 quasi-synchronous and 11 synchronous) were revealed between the electrodes (that is, across assemblies). The proportion of local connections $(0.19)$ was found to be significantly higher $(z$-test, $P<0.00001)$ than the proportion of distal connections (0.02) which seems to be in line with a report (Fujisawa et al., 2008), wherein, authors have reported that connection-probability (number of connections) declines as a function of distance between recorded neurons. Collectively, this suggests that predominantly the interactions between neurons occur locally.

\section{Interaction between the regular-spiking (RS) and fast-spiking (FS) neurons}

In cortical circuits, inhibitory neurons are interspersed within networks of excitatory principal neurons (Nowak et al., 2003; Fries et al., 2007; Bopp et al., 2014; Cossell et al., 2015). Thus, we next wondered how these two peculiar classes of neurons might connect with each other within microcircuits. Electrophysiologically, based upon the ascending slope of the waveform, these two types can be dissociated from each other (Nowak et al., 2003; Niell \& Stryker, 2008; Vinck et al., 2013) into RS and FS neurons which are putatively linked to excitatory principal cells and

This article is protected by copyright. All rights reserved. 
inhibitory neurons, respectively. We thus categorized the neurons as RS and FS, wherein waveforms with slopes $\leq 0.3$ were dissociated as FS, whereas waveforms with slopes $>0.3$ were separated as RS. The dissociation is shown in Fig. 10A (FS, $\mathrm{n}=50$ and RS, $\mathrm{n}=124$; a typical example of an RS and an FS is shown superimposed as an inset). Thereafter, we investigated the connections among these two classes (i.e., RS-RS, the connections between regular spiking neurons; RS-FS, the connections between regular- and fast-spiking neurons; FS-FS, the connections between fast spiking neurons). The proportion of connections declined (Fig. 10B) from RS-RS (0.22) to RS-FS (0.17) to FS-FS (0.12) and each of the classes was significantly different from each other ( $z$-test, between RS-RS and RS-FS, $P=0.005$; between RS-RS and FSFS, $P=0.0002$; between RS-FS and FS-FS, z-test, $P=0.034)$.

Further, we labeled these interactions as excitatory or inhibitory depending upon the position of peaks or troughs in CCGs (Barthó et al., 2004; Schwindel et al., 2014). Out of the proportion of RS-RS interactions, 134 were deemed unidirectional (excitatory in one direction, e.g. connection between neuron 4 and neuron 5 in Fig. 3) and 48 were identified as bidirectional (recurrent excitation, e.g. connection between neurons 1 and 4 in Fig. 3). Among FS-RS interactions, four major types of interactions occurred: inhibitory (FS to RS, unidirectional, $n=27$ ); inhibitoryexcitatory (FS to RS, bidirectional, $\mathrm{n}=18$ ); excitatory ( $\mathrm{FS}$ to $\mathrm{RS}$, unidirectional, $\mathrm{n}=22$ ) and excitatory (RS to FS, unidirectional, $\mathrm{n}=33$ ). A typical example of an inhibitory connection is shown in Fig. 11A. The reference neuron (FS, orange) completely suppressed the activity of the target neuron (RS, target) for few milliseconds $(\sim 5 \mathrm{~ms})$ after the zero point in the CCG. A typical example of inhibitory-excitatory connection is illustrated between neuron 3 (identified as FS, reference) and neuron 1 (RS) in Fig 4. Notice the strong suppression of the RS neuron at zero

This article is protected by copyright. All rights reserved. 
point in the CCG. This indicates the inhibition of RS neuron in one direction and excitation of FS neuron in another direction (Barthó et al., 2004). An example of an FS neuron exciting an RS neuron is signified by neuron 3 to neuron 2 in Fig. 4. In a similar fashion, RS neuron projected onto FS neuron. Finally, among FS-FS interactions, 20 were marked as unidirectional (Fig. 11B, the orange projected onto the brown neuron) and 5 were identified as bidirectional (Fig. 11C, the orange neuron and the blue neuron reciprocally excited each other). The modulation of connections with orientation is displayed in Fig 5.

In summary, this analysis indicates that in cat primary visual microcircuits, the interactions between regular spiking cells (RS-RS) are predominant, which putatively maybe attributed to the principal excitatory neurons. The FS neurons target a few RS neurons in cat visual cortex, unlike the mouse cortices where RS neurons weave on average more FS neurons. This seems in line with earlier reports (Hofer et al., 2011; Bopp et al., 2014; Cossell et al., 2015) and these interactions point to the role of FSs in maintaining the orientation selectivity of RS neurons through distinct mechanisms (Tiesinga \& Sejnowski, 2009) . However, it is to be underlined that in cat visual cortex, the fool-proof separation of spikes into putative pyramidal and inhibitory neurons based on ascending slopes is not entirely possible as a fraction of excitatory neurons may exhibit FS pattern (Nowak et al., 2003). Therefore, one has to be cautious while interpreting the above analysis, as this may not exhaustively suggest inhibitory and excitatory connections between these two classes of neurons in cat primary visual cortex.

This article is protected by copyright. All rights reserved. 


\section{Similarity Pattern of Cross-correlograms (Network stability)}

To ascertain that the revealed functional connections are indeed an outcome of specific coordinated activity between neurons rather than the result of random fluctuations, some neuron pairs recorded simultaneously were tested two times; that is, a particular test feature presentation was repeated after almost an hour. Two recordings were tested twice, i.e., four sites. Fourteen neurons were sorted from these recordings. An example of two simultaneously recorded neurons from a tip (red and blue waveforms) with their respective responses (raster and PSTH) at both attempts is shown in Fig. 12A. The black arrow pointing downwards indicates the presentation of stimulus. The CCG at each attempt in Fig. 12B points that the red neuron projects onto the blue neuron in either case with noticeable similarity. In both attempts, the significant peak appeared at the same time (+ $4 \mathrm{~ms}$, green line) with $\mathrm{P}$ equaling $1.4 \%$. An example of network that remains similar (same connections with similar P) in both attempts between three simultaneously recorded neurons is shown in Fig. 12C. A few connected neuron pairs $(n=26)$ were pooled, and no significant difference (paired $t$-test, $P=0.24$ ) was found between the peak-probability at both attempts (Fig. 12D). This suggests that neurons establish specific functional connections in relation to a particular input that remain constant over time within an assembly, as suggested by Miller et al (2014).

\section{Discussion}

We performed multi-electrode recordings to capture V1 assemblies in cats. The goal was to examine functional connectivity (by employing CCG) between neurons in an assembly in response to different orientation gratings. The main findings of the current investigation are as follows. First, and most important, we found that an assembly structures a salient functional 
network at every orientation. Second, in addition to the closely tuned assemblies, high orientation-range assemblies also featured stimulus-encoding networks between themselves; however, within these assemblies, closely tuned neurons were connected more frequently and vigorously than distantly tuned neurons; untuned neurons did not exhibit many connections. Third, the connections between the RS neurons dominated the connections between RS-FS, and FS-FS neurons. Fourth, the functional connections in assemblies remain stable over a period of time. Fifth, despite low firing, neurons displayed connections.

\section{Methodological Considerations}

The recordings were done in anesthetized cats; therefore, the neuronal output cannot be attributed to the attention parameters (de Kock \& Sakmann, 2009). Spontaneous oscillations could induce time locked neural activity in an anesthetized animal (Poulet \& Petersen, 2008); however, rhythmic oscillations that permit neuronal synchrony can also be recorded in anesthetized state (Gray \& Singer, 1989; Xing et al., 2012; Bharmauria et al., 2015). In current experiments, the stimuli were applied in a random fashion with random inter-stimulus intervals. The assemblies exhibited more connections when visually evoked; the connectivity and connection-strength decreased as a function of orientation spread as expected (Hofer et al., 2011; Ko et al., 2011; Denman \& Contreras, 2014); the interactions between neurons were predominantly local and the pattern of CCGs remained similar with time. Moreover, we have already documented that connected neurons in an assembly cooperate synergistically than their unconnected companions within a characteristical 50-ms window of opportunity exhibiting heightened gamma power (Bharmauria et al., 2014; Bharmauria et al., 2015). All these

This article is protected by copyright. All rights reserved. 
significant trends along with our previous findings suggest that the divulged functional connections were indeed strongly linked to the input stimulus.

\section{Functional network: the encoding signature of an assembly}

Many previous studies using different approaches such as calcium imaging (Hofer et al., 2011; Miller et al., 2014; Cossell et al., 2015; Wertz et al., 2015), electrophysiology (Barthó et al., 2004; Fujisawa et al., 2008; Ecker et al., 2010; Denman \& Contreras, 2014), and computational modeling (Binzegger et al., 2004; Buzas et al., 2006) have pointed to the significance of specific local connectivity in neural circuits; however none of them explored the functional interactions in an assembly to the systematic presentation of a stimulus, as in this investigation (the systematic tilt of the oriented gratings). We found that a salient network is activated in an assembly at each orientation, wherein, connections are activated or inactivated depending on the feature. To draw an analogy with Singer's postulation (Singer, 2013) - a reservoir (assembly in our case) recruits a specific classifier (network) within its randomly coupled neurons contingent upon the input. Moreover, some connections are frequently active as if embedded strongly in an assembly irrespective of the input. In addition, new cells might become active (start responding) and some may leave the assembly in relation to the grating. Along with previous reports (Barth \& Poulet, 2012; Cossell et al., 2015), we suggest that, in an assembly there is a core group of active cells that interact synergistically (Bharmauria et al., 2014) and may always remain connected, whereas the weakly connected neurons confer flexibility to the assembly as the input changes. In addition, the assemblies were active intrinsically (absence of input) too, as has been reported previously (Kenet et al., 2003; Han et al., 2008; Mohajerani et al., 2013; Miller et al., 2014), but did not display many connections in comparison to the visual stimulation. This

This article is protected by copyright. All rights reserved. 
reverberating activity maybe a fundamental precursor for the organization of neural circuits that develops with past experience (during youth and adulthood), thereby, keeping the circuit always prepared to receive the input efficiently. Any relevant input may then propel intrinsically active circuit into a biased functional mode (Miller et al., 2014). Lastly, the stability of connections with time reflects upon the specific time-locked neural activities in an assembly in relation to the same stimulus. Conclusively, all above findings and arguments imply that a salient functional network within an emergent assembly is its 'encoding signature' to a particular input.

\section{Firing rate of neurons, functional interactions and their strength}

Neuronal selectivity (implying the firing rate) is the classical method to study the feature encoding (e.g., in V1 neurons), and sparse firing is a characteristic feature of microcircuits (Barth \& Poulet, 2012; Jayakumar et al., 2012; Molotchnikoff \& Rouat, 2012). We found that significant peaks (and their probabilities) in CCGs may emerge irrespective of the low firing of the involved pair. Thus, it can be inferred that connectivity between low firing neurons (and at non-optimal orientations) is attributed to the precise spike timing of the reference neuron to modulate the firing of the recipient cell. Indeed, several arguments militate against a possible bias of firing rates for significant CCGs (Duret et al., 2006; Ghisovan et al., 2008; Rolls \&

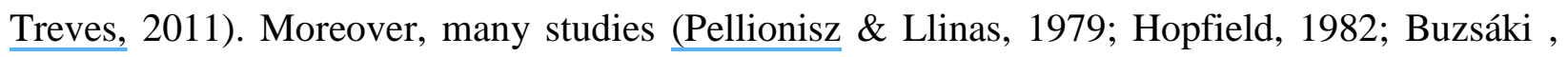
2010; Miller et al., 2014) coincide to the conclusion that, in an assembly of interconnected neurons, the firing rate of an individual neuron is not as important as the whole network. Lastly, it has been shown that population responses decode the tasks better than individual neurons (as the response of a neurons is promiscuous even to the same stimulus) (Kampa et al., 2011). One may also argue that the brain state of an anesthetized animal might control the number of

This article is protected by copyright. All rights reserved. 
responsive neurons and suppress the response output (de Kock \& Sakmann, 2009). Thus, timelocked events between neurons might go undetected due to low firing of neurons or insufficient trials (stimulus presentation) that may result in few spikes for CCG analysis. The number of trials and their duration may change from one experimental setup to another. In our case, the duration of stimulation was relatively long to collect enough spikes for CCG analysis; nevertheless, the possibility of some undetected connections cannot be eliminated. Indeed, the equivalent firing of connected and unconnected neurons (Fig. 6C) underlines the importance of precisely locked firing between neurons than the firing rate of neurons. The strongly embedded connections in circuits may consistently show such time-locked neural activities (Cossell et al., 2015). In summary, we suggest that an assembly (and thus the activated network) is more important than its neurons as separate individuals, wherein the weakly firing neurons may also play an important role in stimulus encoding.

\section{The importance of neuronal selectivity and RS-FS interactions}

We found that the closely tuned neurons exhibited increased connectivity and connection strengths than the distantly tuned neurons within assemblies. This is in line with previous reports (Gilbert \& Wiesel, 1989; Shadlen \& Newsome, 1998; Kisvárday et al., 2000; Stepanyants et al., 2008; de Kock \& Sakmann, 2009; Hofer et al., 2011; Ko et al., 2011; Ko et al., 2013; Wertz et al., 2015; Ecker et al., 2010), as neurons with similar response properties densely innervate each other, have correlated spike activities, and receive a common peripheral input, therefore, having a maximal likelihood of being connected. This suggests that the network activity is biased towards a particular range of close orientation. The similarly tuned neurons encode the similar features in a target, whereas the differentially or oppositely tuned pairs may simultaneously bind

This article is protected by copyright. All rights reserved. 
the contrasting or opposing features, respectively, to establish the entirety of a complex target (Singer, 2001; Molotchnikoff \& Rouat, 2012). Because closely-tuned neurons have simultaneous high firing rates, such occurrence may allow a reduction of the overall space of possible trajectories or operating connections; otherwise, the population would not exhibit networkselectivity. The subset of neurons most closely tuned to a stimulus may thus drive the activity of the connected cell within the network, producing stable neuronal trajectories and networkselectivity. On the other hand, in a higher orientation spread, we are likely to observe more contrast between the firing rates eventually constraining the circuit to a limited number of functional connections. Lastly, the untuned neurons exhibited a few connections, implying that neuronal selectivity is an essential determinant of network-selection.

Finally, the predominance of RS-RS interactions alludes to the importance of recurrent excitatory connections within V1 assemblies between these particular cells, wherein, FS neurons calibrate the selectivity of RS neurons (Hofer et al., 2011; Bopp et al., 2014; Cossell et al., 2015).

\section{Conclusion}

In current experiments, the neurons comprising assemblies were relatively few in comparison to the suggested size of population-codes (Alivisatoset al., 2012). Indeed, the striking specificity of interacting neurons from one orientation to another reflects upon the temporal signature and dynamics of a stimulus-encoding assembly. This study further establishes the significance of cell-assembly as the main functional unit of information processing in cortical circuits and dilutes the importance of an individually firing neuron in encoding the stimulus. In short, this

This article is protected by copyright. All rights reserved. 
work could be a step toward the further unraveling of the neuronal codes governing cortical responses.

\section{Competing Interests}

The authors declare no competing financial interests.

\section{Acknowledgements}

SM and JR were supported by CRSNG (Conseil de Recherches en Sciences Naturelles et en Génie) FRQ-NT (Fonds de recherche du Québec - Nature et technologies). SB was supported by a CRSNG Graduate scholarship. Grant No: NSERC, Canada 6943-1210.

\section{Abbreviations}

ACG, auto-correlogram, CCG, cross-correlogram; FS, fast spike; OSI, orientation selectivity index; PCA, principal component analysis; RF, receptive field; RS, regular spike; SEM, standard error of the mean; V1, primary visual cortex

\section{Author Contributions}

VB did the experiments, analyzed the data and wrote the manuscript. LB, SC and NC participated in experiments and analyses of data. SB and JR contributed to the analyses of data. VB and SM conceived the idea of study and contributed to data analyses and manuscript writing.

This article is protected by copyright. All rights reserved. 


\section{References}

Abeles, M. (1982) Quantification, smoothing, and confidence limits for single-units' histograms. J. Neurosci. Methods, 5, 317-325.

Alivisatos, A.P., Chun, M., Church, G.M., Greenspan, R.J., Roukes, M.L. \& Yuste, R. (2012) The brain activity map project and the challenge of functional connectomics. Neuron, $\mathbf{7 4}$, 970-974.

Alloway, K.D. \& Roy, S.A. (2002) Conditional cross-correlation analysis of thalamocortical neurotransmission. Behav. Brain Res., 135, 191-196.

Bachatene, L., Bharmauria, V., Cattan, S. \& Molotchnikoff, S. (2013) Fluoxetine and serotonin facilitate attractive-adaptation-induced orientation plasticity in adult cat visual cortex. Euro. J. Neurosci., 38, 2065-2077.

Bardy, C., Huang, J.Y., Wang, C., FitzGibbon, T. \& Dreher, B. (2006) 'Simplification' of responses of complex cells in cat striate cortex: suppressive surrounds and 'feedback' inactivation. J. Physiol., 574, 731-750.

Barlow, H.B., Blakemore, C. \& Pettigrew, J.D. (1967) The neural mechanism of binocular depth discrimination. J. Physiol., 193, 327-342.

Barth, A.L. \& Poulet, J.F. (2012) Experimental evidence for sparse firing in the neocortex. Trends Neurosci., 35, 345-355.

Barthó, P., Hirase, H., Monconduit, L., Zugaro, M., Harris, K.D. \&Buzsáki , G. (2004) Characterization of neocortical principal cells and interneurons by network interactions and extracellular features. J. Neurophysiol., 92, 600-608.

Bharmauria, V., Bachatene, L., Cattan, S., Chanauria, N., Rouat, J. \& Molotchnikoff, S. (2015) Stimulus-dependent augmented gamma oscillatory activity between the functionally connected cortical neurons in the primary visual cortex. Euro. J. Neurosci., 41, 15871596.

Bharmauria, V., Bachatene, L., Cattan, S., Rouat, J. \& Molotchnikoff, S. (2014) Synergistic activity between primary visual neurons. Neuroscience, 268, 255-264.

Binzegger, T., Douglas, R.J. \& Martin, K.A. (2004) A quantitative map of the circuit of cat primary visual cortex. J. Neurosci., 24, 8441-8453.

Bishop, P.O. \& Henry, G.H. (1972) Striate neurons: receptive field concepts. Invest. Ophthalmol., 11, 346-354.

Bopp, R., Macarico da Costa, N., Kampa, B.M., Martin, K.A. \& Roth, M.M. (2014) Pyramidal cells make specific connections onto smooth (GABAergic) neurons in mouse visual

This article is protected by copyright. All rights reserved. 
cortex. PLoS Biol., 12, e1001932.

Butts, D.A. \& Goldman, M.S. (2006) Tuning curves, neuronal variability, and sensory coding. PLoS Biol., 4, e92.

Buzas, P., Kovacs, K., Ferecsko, A.S., Budd, J.M., Eysel, U.T. \&Kisvárday, Z.F. (2006) Modelbased analysis of excitatory lateral connections in the visual cortex. J. Comput. Neurosci., 499, 861-881.

Buzsáki , G. (2010) Neural syntax: cell assemblies, synapsembles, and readers. Neuron, 68, 362385.

Cossell, L., Iacaruso, M.F., Muir, D.R., Houlton, R., Sader, E.N., Ko, H., Hofer, S.B. \& MrsicFlogel, T.D. (2015) Functional organization of excitatory synaptic strength in primary visual cortex. Nature, 518, 399-403.

Csicsvari, J., Hirase, H., Czurko, A. \&Buzsáki , G. (1998) Reliability and state dependence of pyramidal cell-interneuron synapses in the hippocampus: an ensemble approach in the behaving rat. Neuron, 21, 179-189.

de Kock, C.P. \& Sakmann, B. (2009) Spiking in primary somatosensory cortex during natural whisking in awake head-restrained rats is cell-type specific. Proc. Natl. Acad. Sci. USA., 106, 16446-16450.

DeAngelis, G.C., Ghose, G.M., Ohzawa, I. \& Freeman, R.D. (1999) Functional microorganization of primary visual cortex: receptive field analysis of nearby neurons. $J$. Neurosci.,19,4046-4064.

Denman, D.J. \& Contreras, D. (2014) The structure of pairwise correlation in mouse primary visual cortex reveals functional organization in the absence of an orientation map. Cereb. Cortex., 24, 2707-2720.

Duret, F., Shumikhina, S. \& Molotchnikoff, S. (2006) Neuron participation in a synchronyencoding assembly. BMC Neurosci., 7, 72.

Ecker, A.S., Berens, P., Keliris, G.A., Bethge, M., Logothetis, N.K. \& Tolias, A.S. (2010) Decorrelated Neuronal Firing in Cortical Microcircuits. Science, 327, 584-587.

Engel, A.K., Fries, P. \& Singer, W. (2001) Dynamic predictions: oscillations and synchrony in top-down processing. Nat. Rev. Neurosci., 2, 704-716.

Fries, P., Nikolic, D. \& Singer, W. (2007) The gamma cycle. Trends Neurosci., 30, 309-316.

Fujisawa, S., Amarasingham, A., Harrison, M.T. \&Buzsáki , G. (2008) Behavior-dependent short-term assembly dynamics in the medial prefrontal cortex. Nat. Neurosci., 11, 823833.

This article is protected by copyright. All rights reserved. 
Ghisovan, N., Nemri, A., Shumikhina, S. \& Molotchnikoff, S. (2008) Synchrony between orientation-selective neurons is modulated during adaptation-induced plasticity in cat visual cortex. BMC Neurosci., 9, 60.

Gilbert, C.D. \& Wiesel, T.N. (1989) Columnar specificity of intrinsic horizontal and corticocortical connections in cat visual cortex. J. Neurosci., 9, 2432-2442.

Gray, C.M. \& Singer, W. (1989) Stimulus-specific neuronal oscillations in orientation columns of cat visual cortex. Proc. Natl. Acad. Sci. USA., 86, 1698-1702.

Hagmann, P., Cammoun, L., Gigandet, X., Gerhard, S., Grant, P.E., Wedeen, V., Meuli, R., Thiran, J.P., Honey, C.J. \& Sporns, O. (2010) MR connectomics: Principles and challenges. J. Neurosci. Methods., 194, 34-45.

Han, F., Caporale, N. \& Dan, Y. (2008) Reverberation of recent visual experience in spontaneous cortical waves. Neuron, 60, 321-327.

Harris, K.D., Hirase, H., Leinekugel, X., Henze, D.A. \&Buzsáki , G. (2001) Temporal interaction between single spikes and complex spike bursts in hippocampal pyramidal cells. Neuron, 32, 141-149.

Harris, K.D. \& Mrsic-Flogel, T.D. (2013) Cortical connectivity and sensory coding. Nature, 503, 51-58.

Hofer, S.B., Ko, H., Pichler, B., Vogelstein, J., Ros, H., Zeng, H., Lein, E., Lesica, N.A. \& Mrsic-Flogel, T.D. (2011) Differential connectivity and response dynamics of excitatory and inhibitory neurons in visual cortex. Nat. Neurosci., 14, 1045-1052.

Hopfield, J.J. (1982) Neural networks and physical systems with emergent collective computational abilities. Proc. Natl. Acad. Sci. USA., 79, 2554-2558.

Hubel, D.H. \& Wiesel, T.N. (1959) Receptive fields of single neurones in the cat's striate cortex. J. Physiol., 148, 574-591.

Jayakumar, J., Hu, D. \& Vidyasagar, T.R. (2012) Sparseness of coding in area 17 of the cat visual cortex: a comparison between pinwheel centres and orientation domains. Neuroscience, 225, 55-64.

Jia, H., Rochefort, N.L., Chen, X. \& Konnerth, A. (2010) Dendritic organization of sensory input to cortical neurons in vivo. Nature, 464, 1307-1312.

Kampa, B.M., Roth, M.M., Gobel, W. \& Helmchen, F. (2011) Representation of visual scenes by local neuronal populations in layer $2 / 3$ of mouse visual cortex. Front. Neural Circuits, $\mathbf{5}$, 18.

This article is protected by copyright. All rights reserved. 
Kenet, T., Bibitchkov, D., Tsodyks, M., Grinvald, A. \& Arieli, A. (2003) Spontaneously emerging cortical representations of visual attributes. Nature, 425, 954-956.

Kisvárday, Z.F., Crook, J.M., Buzas, P. \& Eysel, U.T. (2000) Combined physiologicalanatomical approaches to study lateral inhibition. J. Neurosci. Methods, 103, 91-106.

Ko, H., Cossell, L., Baragli, C., Antolik, J., Clopath, C., Hofer, S.B. \& Mrsic-Flogel, T.D. (2013) The emergence of functional microcircuits in visual cortex. Nature, 496, 96-100.

Ko, H., Hofer, S.B., Pichler, B., Buchanan, K.A., Sjostrom, P.J. \& Mrsic-Flogel, T.D. (2011) Functional specificity of local synaptic connections in neocortical networks. Nature, 473, 87-91.

König, P., Engel, K., Roelfsema, P.R.\& Singer, W. (1995) How precise is the neuronal synchronization? Neural Comput.,7,469-485.

Leergaard, T.B., Hilgetag, C.C. \& Sporns, O. (2012) Mapping the connectome: multi-level analysis of brain connectivity. Front. Neuroinform., 6, 14.

Liao, D.S., Krahe, T.E., Prusky, G.T., Medina, A.E. \& Ramoa, A.S. (2004) Recovery of cortical binocularity and orientation selectivity after the critical period for ocular dominance plasticity. J. Neurophysiol., 92, 2113-2121.

Martin, K.A. \&Schröder, S. (2013) Functional heterogeneity in neighboring neurons of cat primary visual cortex in response to both artificial and natural stimuli. J. Neurosci.,33, 7325-7344.

Miller, J.E., Ayzenshtat, I., Carrillo-Reid, L. \& Yuste, R. (2014) Visual stimuli recruit intrinsically generated cortical ensembles. Proc. Natl. Acad. Sci. USA., 111, E4053-4061.

Mohajerani, M.H., Chan, A.W., Mohsenvand, M., LeDue, J., Liu, R., McVea, D.A., Boyd, J.D., Wang, Y.T., Reimers, M. \& Murphy, T.H. (2013) Spontaneous cortical activity alternates between motifs defined by regional axonal projections. Nat. Neurosci., 16, 1426-1435.

Molotchnikoff, S. \& Rouat, J. (2012) Brain at work: time, sparseness and superposition principles. Front. Biosci., 17, 583-606.

Niell, C.M. \& Stryker, M.P. (2008) Highly selective receptive fields in mouse visual cortex. $J$. Neurosci., 28, 7520-7536.

Nowak, L.G., Azouz, R., Sanchez-Vives, M.V., Gray, C.M. \& McCormick, D.A. (2003) Electrophysiological classes of cat primary visual cortical neurons in vivo as revealed by quantitative analyses. J. Neurophysiol., 89, 1541-1566.

Pellionisz, A. \& Llinas, R. (1979) Brain modeling by tensor network theory and computer simulation. The cerebellum: distributed processor for predictive coordination.

This article is protected by copyright. All rights reserved. 
Neuroscience, 4, 323-348.

Perkel, D.H., Gerstein, G.L. \& Moore, G.P. (1967) Neuronal spike trains and stochastic point processes. I. The single spike train. Biophysi. J.,7, 391-418.

Poulet, J.F. \& Petersen, C.C. (2008) Internal brain state regulates membrane potential synchrony in barrel cortex of behaving mice. Nature, 454, 881-885.

Ramoa, A.S., Mower, A.F., Liao, D. \& Jafri, S.I. (2001) Suppression of cortical NMDA receptor function prevents development of orientation selectivity in the primary visual cortex. $J$. Neurosci., 21, 4299-4309.

Rolls, E.T. \& Treves, A. (2011) The neuronal encoding of information in the brain. Prog. Neurobiol., 95, 448-490.

Schwindel, C.D., Ali, K., McNaughton, B.L. \& Tatsuno, M. (2014) Long-term recordings improve the detection of weak excitatory-excitatory connections in rat prefrontal cortex. J. Neurosci., 34, 5454-5467.

Shadlen, M.N. \& Newsome, W.T. (1998) The variable discharge of cortical neurons: implications for connectivity, computation, and information coding. J. Neurosci.,18, 3870-3896.

Shannon, R., Baekey, D.M., Morris, K.F., Li, Z. \& Lindsey, B.G. (2000) Functional connectivity among ventrolateral medullary respiratory neurones and responses during fictive cough in the cat. J. Physiol., 525 Pt 1, 207-224.

Singer, W. (2001) Consciousness and the binding problem. Ann. N. Y. Acad. Sci., 929, 123-146.

Singer, W. (2013) Cortical dynamics revisited. Trends Cogn. Sci., 17, 616-626.

Sporns, O. (2011) The human connectome: a complex network. Ann. N. Y. Acad. Sci.,1224, 109125.

Sporns, O., Tononi, G. \& Kotter, R. (2005) The human connectome: A structural description of the human brain. PLoS Comput. Biol., 1, e42.

Stepanyants, A., Hirsch, J.A., Martinez, L.M., Kisvárday, Z.F., Ferecsko, A.S. \& Chklovskii, D.B. (2008) Local potential connectivity in cat primary visual cortex. Cereb. Cortex., 18, 13-28.

Swindale, N.V. (1998) Orientation tuning curves: empirical description and estimation of parameters. Biol. Cybern., 78, 45-56.

Talebi, V. \& Baker, C.L. (2012) Natural versus synthetic stimuli for estimating Receptive field models: a comparison of predictive robustness. J. Neurosci., 32, 1560-1576.

This article is protected by copyright. All rights reserved. 
Tiesinga, P. \& Sejnowski, T.J. (2009) Cortical Enlightenment: Are attentional gamma oscillations driven by ING or PING? Neuron, 63, 727-732.

Vinck, M., Womelsdorf, T., Buffalo, E.A., Desimone, R. \& Fries, P. (2013) Attentional Modulation of Cell-Class-Specific Gamma-Band Synchronization in Awake Monkey Area V4. Neuron, 80, 1077-1089.

Vizuete, J.A., Pillay, S., Diba, K., Ropella, K.M. \& Hudetz, A.G. (2012) Monosynaptic functional connectivity in cerebral cortex during wakefulness and under graded levels of anesthesia. Front. Integr. Neurosci., 6, 90.

Wertz, A., Trenholm, S., Yonehara, K., Hillier, D., Raics, Z., Leinweber, M., Szalay, G., Ghanem, A., Keller, G., Rozsa, B., Conzelmann, K.K. \& Roska, B. (2015) Single-cellinitiated monosynaptic tracing reveals layer-specific cortical network modules. Science, 349, 70-74.

Xing, D., Shen, Y., Burns, S., Yeh, C.I., Shapley, R. \& Li, W. (2012) Stochastic generation of gamma-band activity in primary visual cortex of awake and anesthetized monkeys. $J$. Neurosci., 32, 13873-13880a

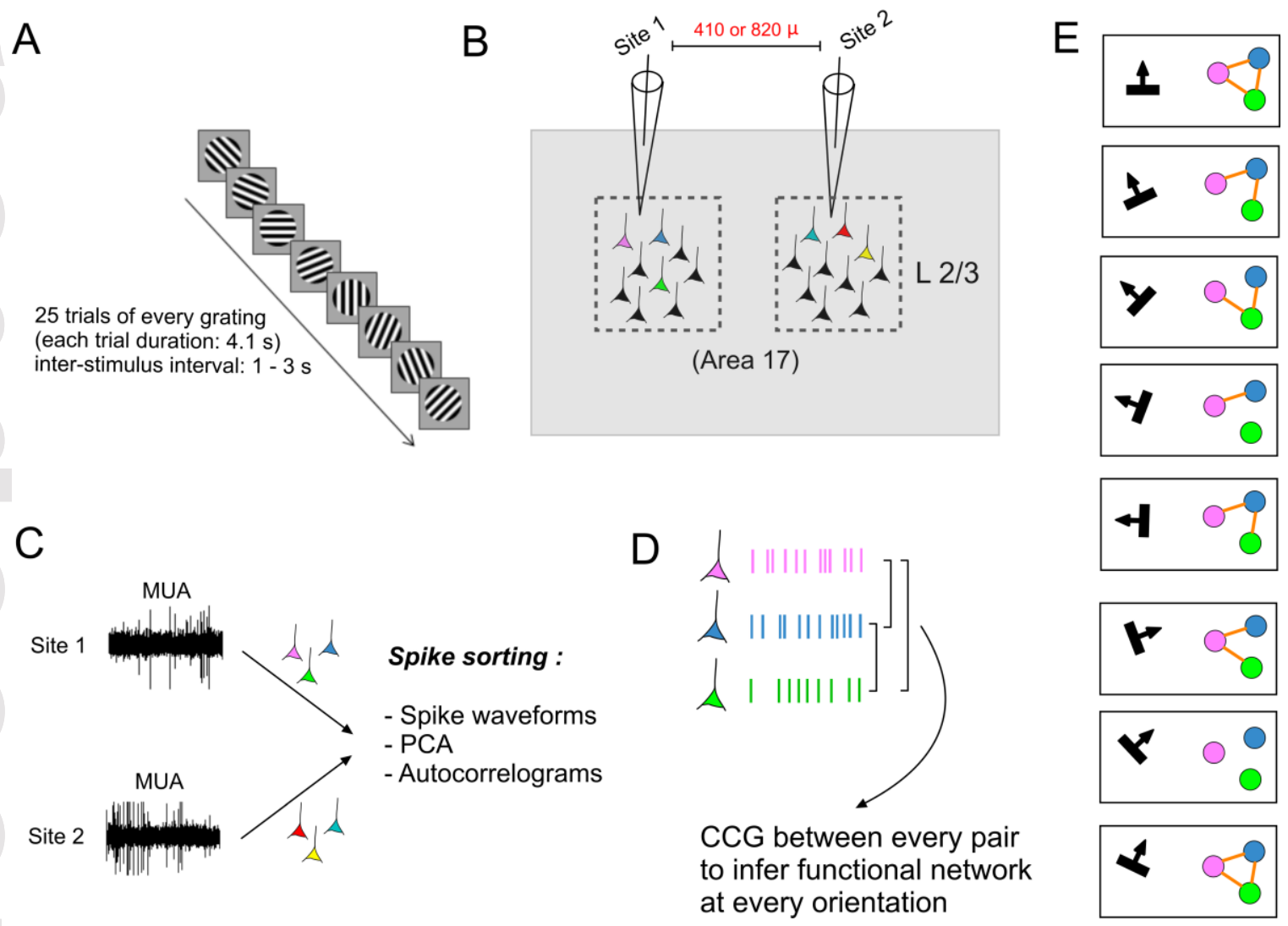

This article is protected by copyright. All rights reserved. 


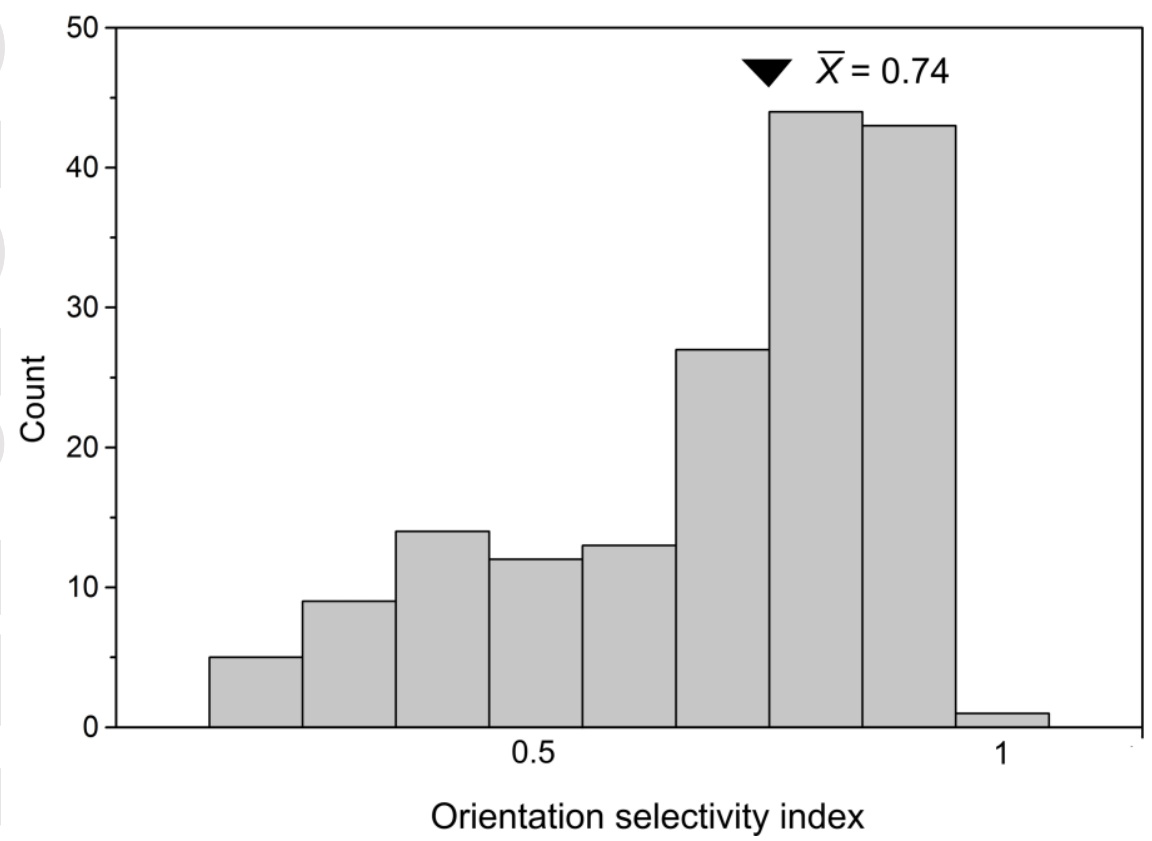

A
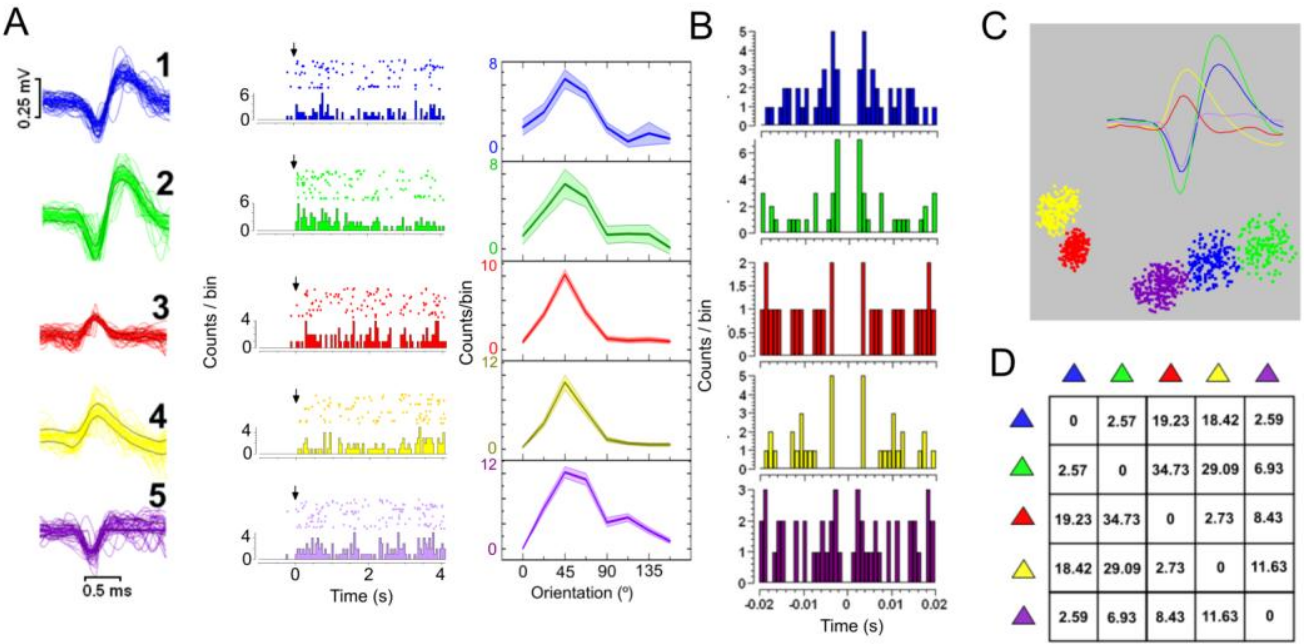

E

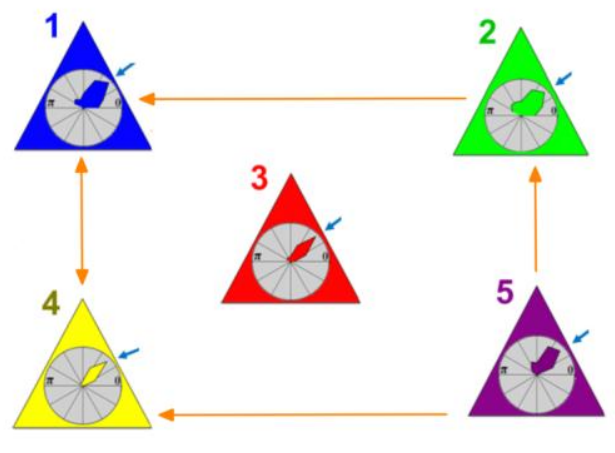

F
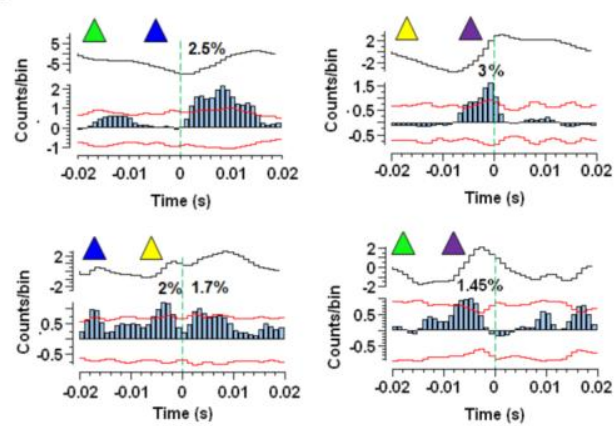

This article is protected by copyright. All rights reserved. 

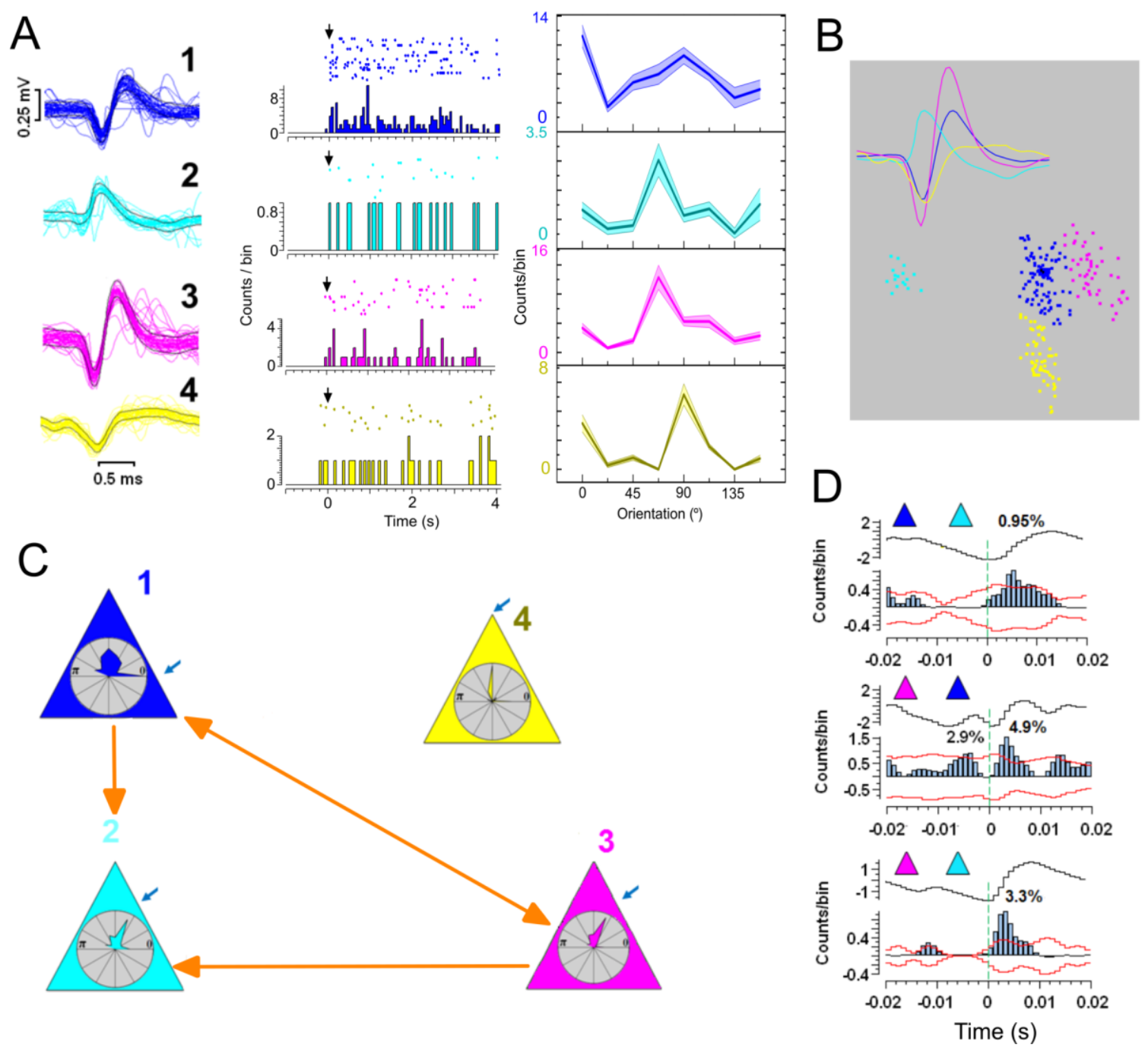

This article is protected by copyright. All rights reserved. 
A
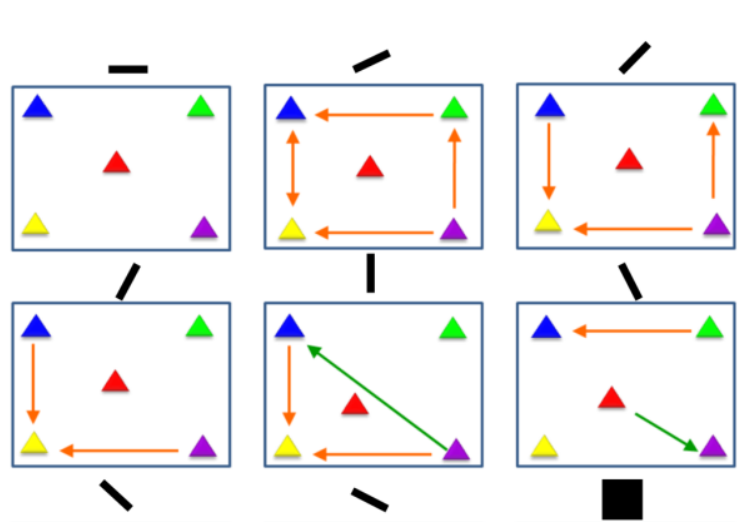

B
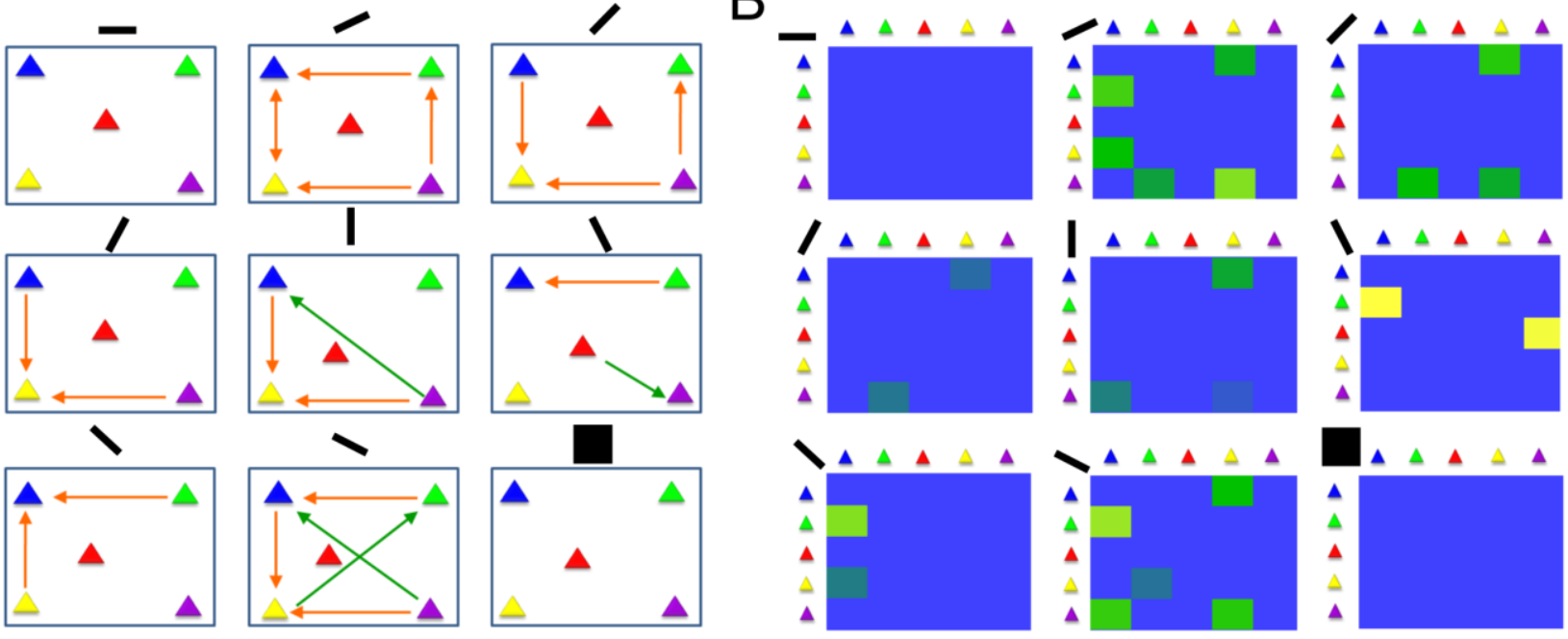

4.1

C

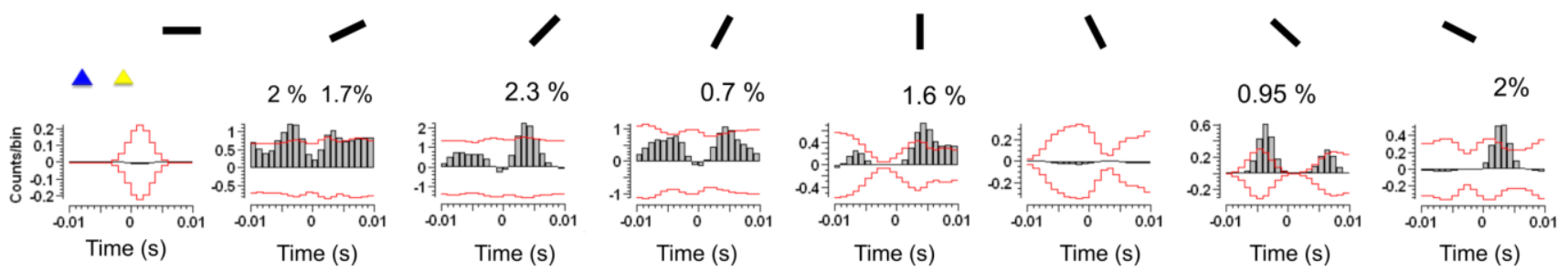

This article is protected by copyright. All rights reserved. 
A $\quad P=0.58$

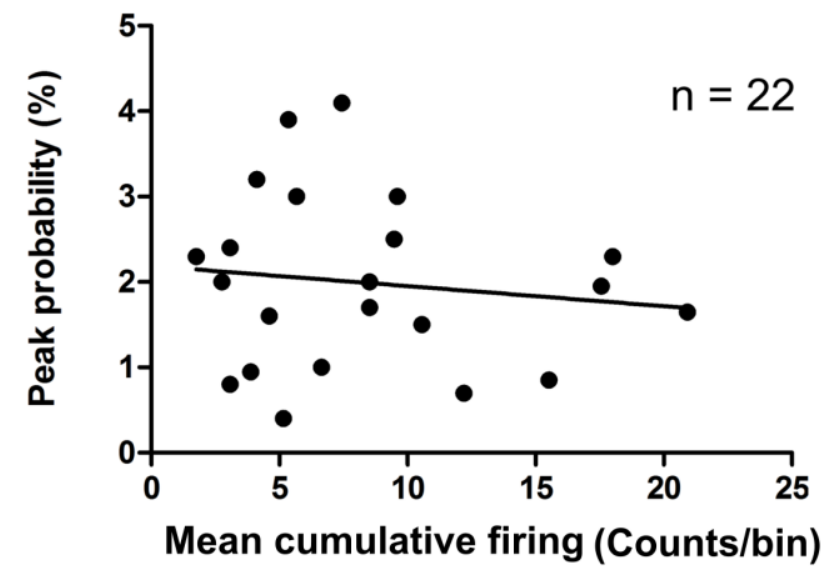

B
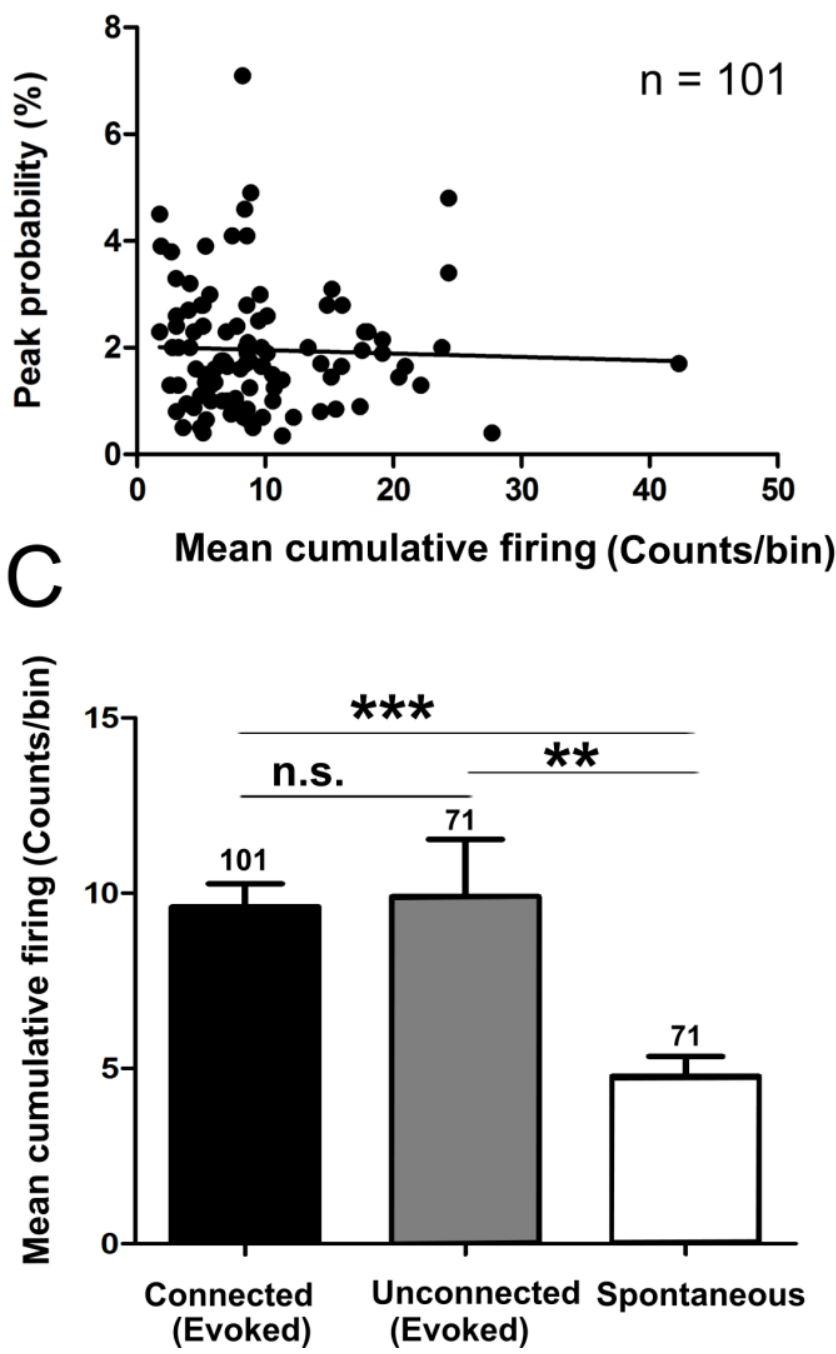

This article is protected by copyright. All rights reserved. 


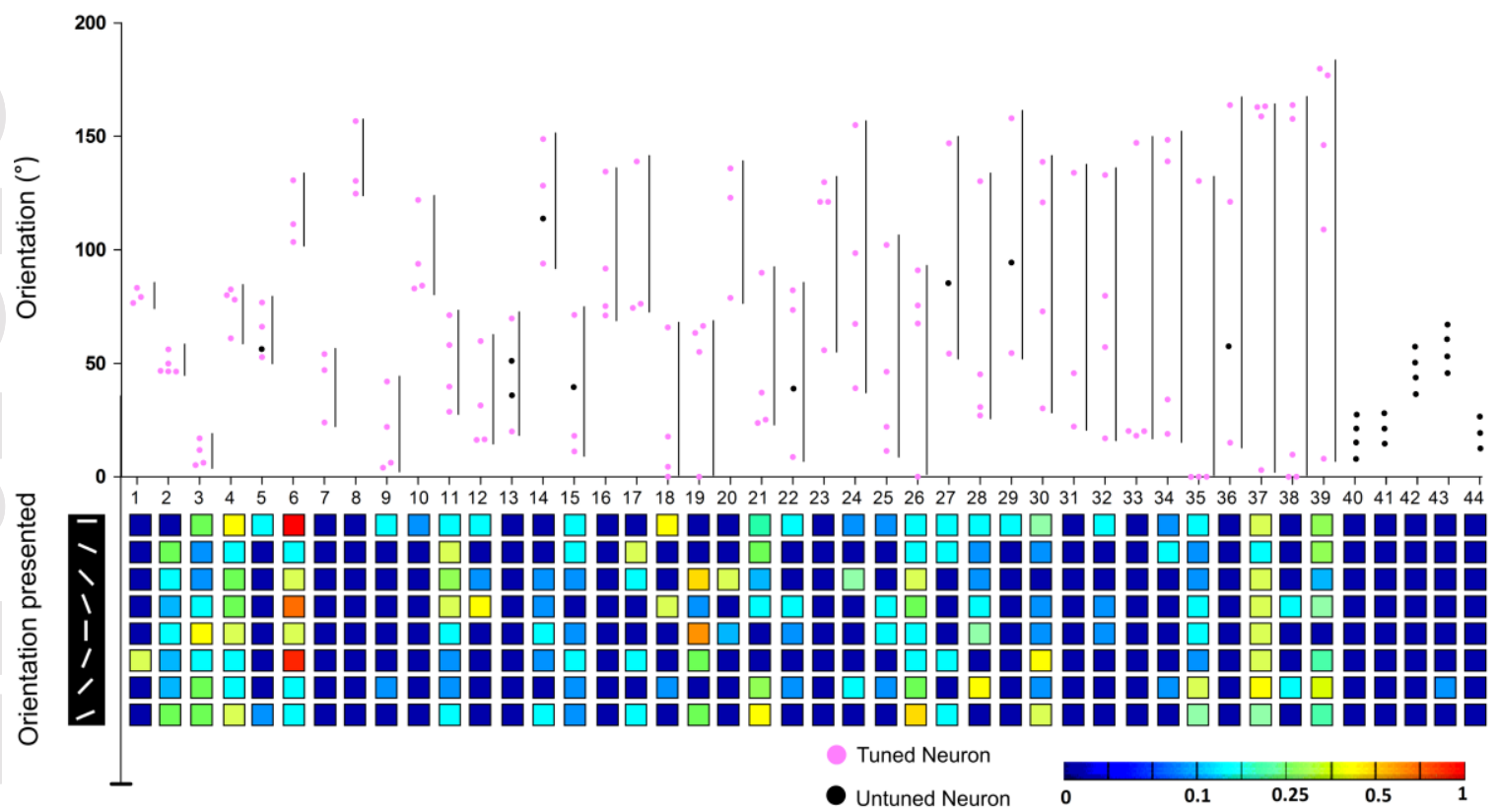

This article is protected by copyright. All rights reserved. 
A

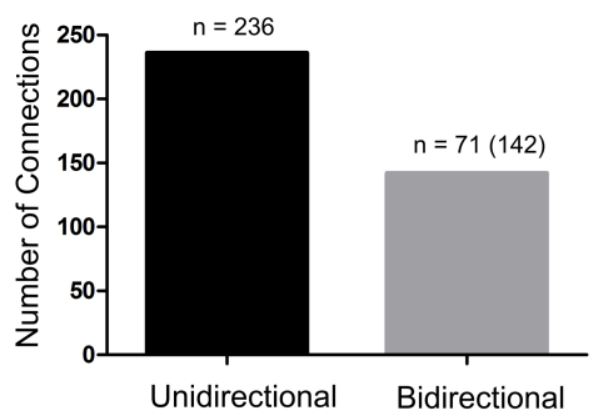

C

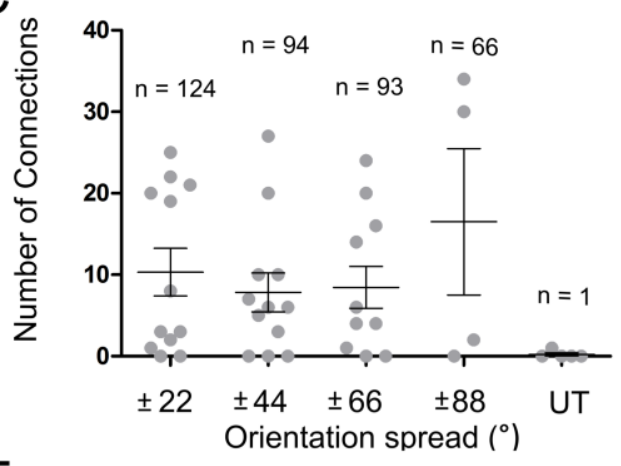

E

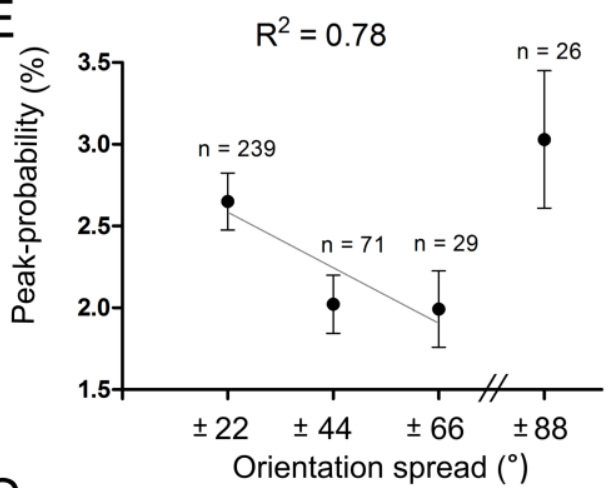

G

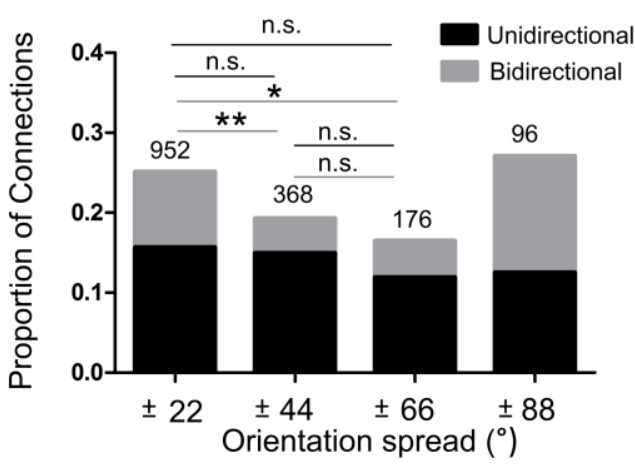

B

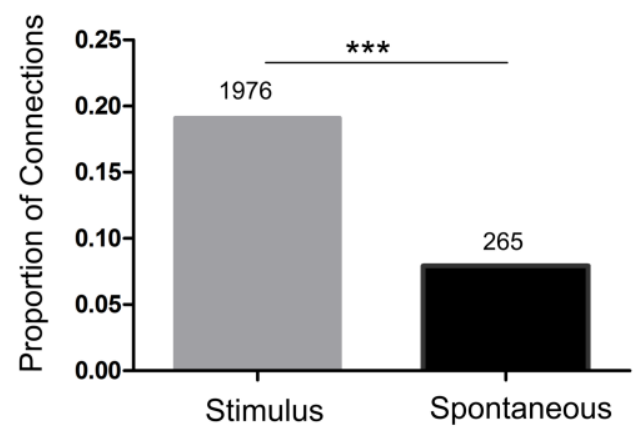

D

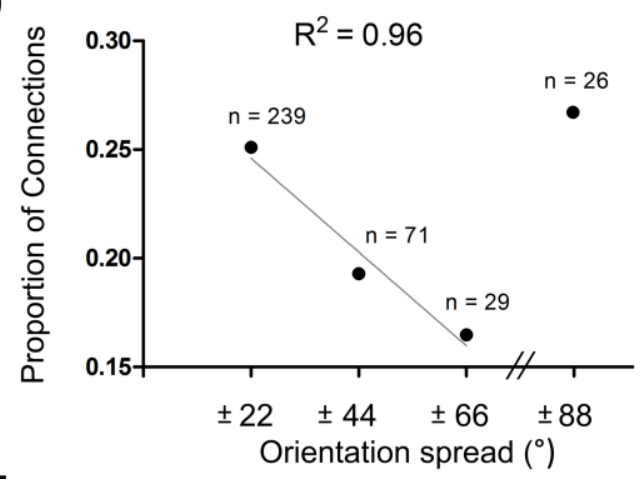

$\mathrm{F}$

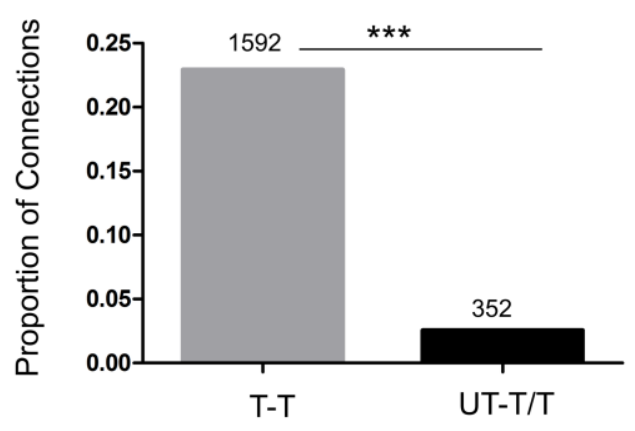

$\mathrm{H}$

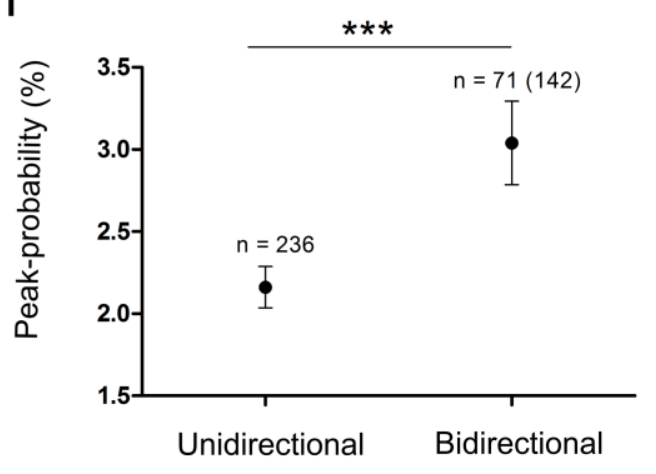

This article is protected by copyright. All rights reserved. 
A

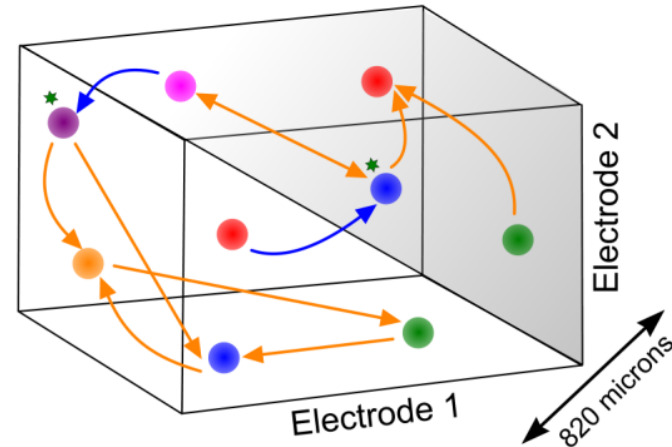

B

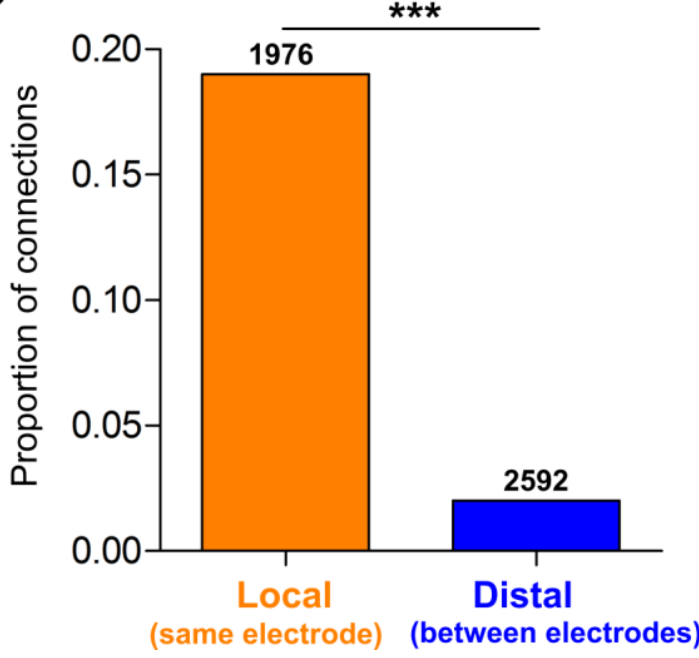

A
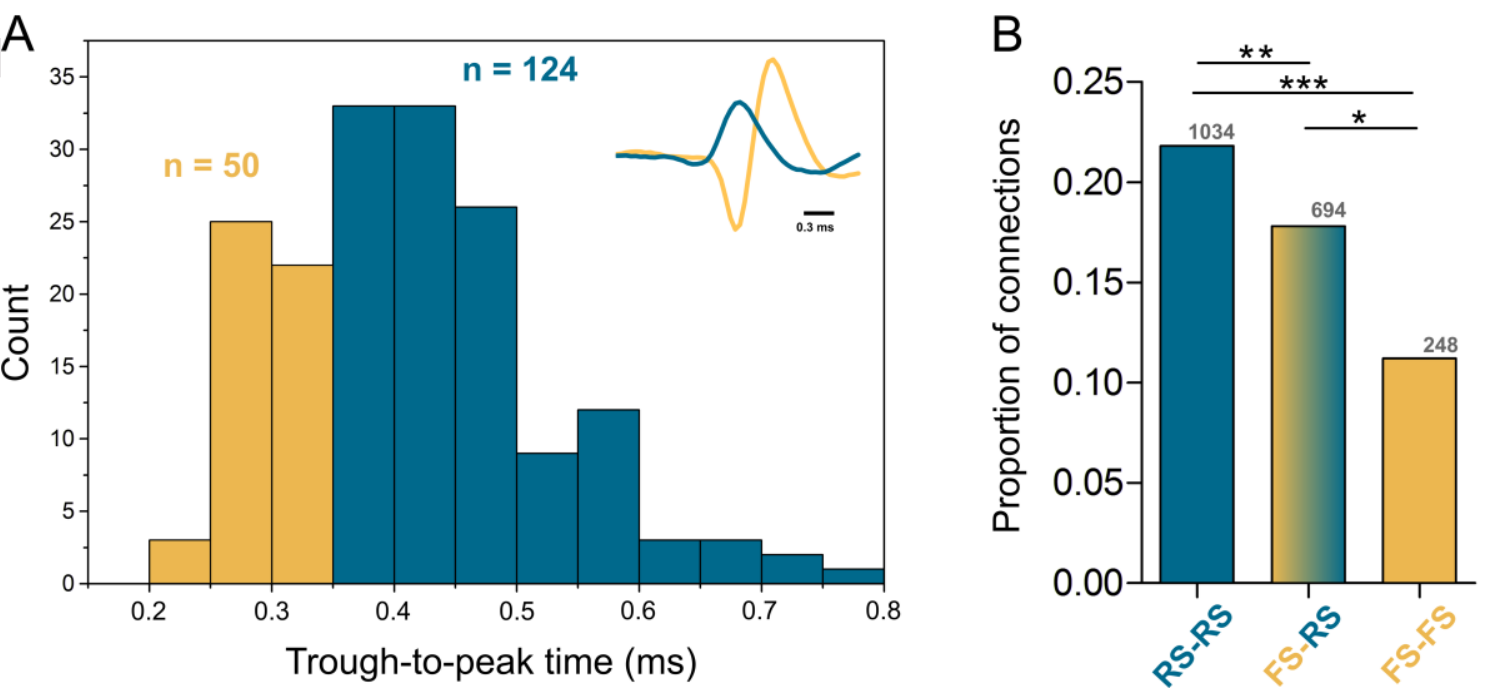

This article is protected by copyright. All rights reserved. 


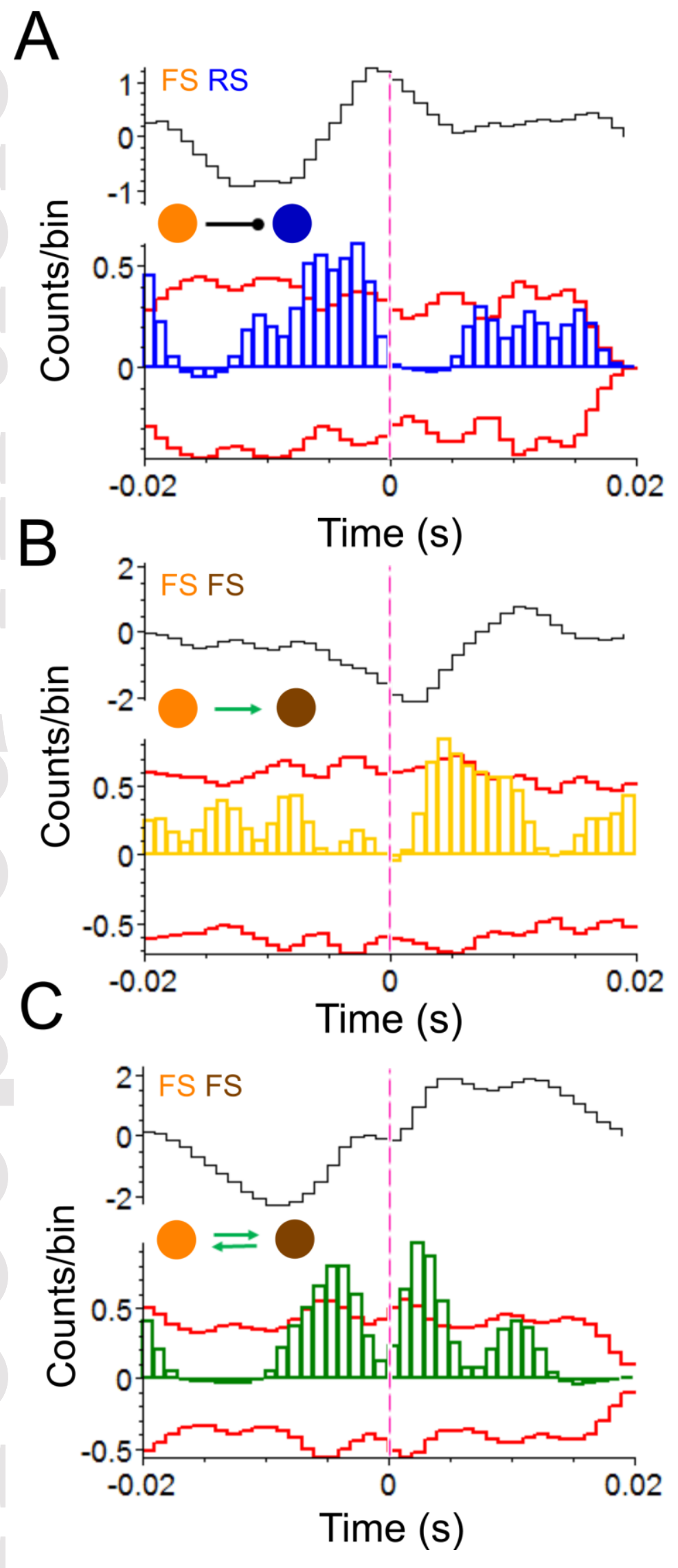

This article is protected by copyright. All rights reserved. 


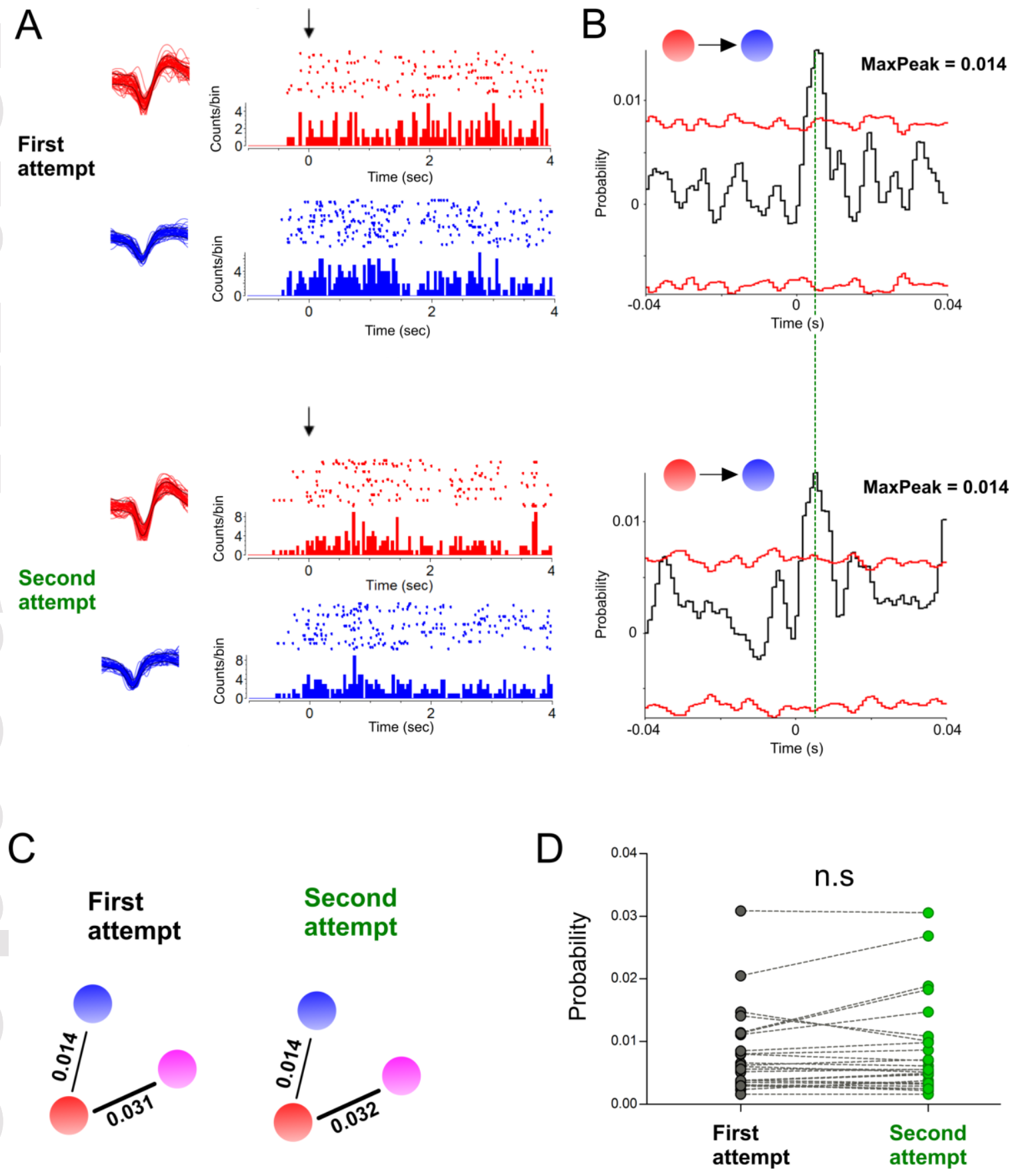

This article is protected by copyright. All rights reserved. 